\title{
Status of global fits to neutrino oscillations
}

\author{
Michele Maltoni†, Thomas Schwetzł, Mariam Tórtola $\S$ and \\ José W. F. Valle§ \\ $\dagger$ C.N. Yang Institute for Theoretical Physics, SUNY at Stony Brook, \\ Stony Brook, NY 11794-3840, USA \\ $\ddagger$ Physik-Department, Technische Universität München, James-Franck-Strasse, \\ D-85748 Garching, Germany \\ $\S$ AHEP Group, Instituto de Física Corpuscular - C.S.I.C./Universitat de València, \\ Edificio Institutos de Paterna, Apt 22085, E-46071 Valencia, Spain \\ E-mail: maltoni@insti.physics.sunysb.edu, schwetz@ph.tum.de, \\ mariam@ific.uv.es, valle@ific.uv.es
}

\begin{abstract}
We review the present status of global analyses of neutrino oscillations, taking into account the most recent neutrino data including the latest KamLAND and K2K updates presented at Neutrino2004, as well as state-of-the-art solar and atmospheric neutrino flux calculations. We give the two-neutrino solar + KamLAND results, and the two-neutrino atmospheric $+\mathrm{K} 2 \mathrm{~K}$ oscillation regions, discussing in each case the robustness of the oscillation interpretation against departures from the Standard Solar Model and the possible existence of non-standard neutrino physics. Furthermore, we give the best fit values and allowed ranges of the three-flavour oscillation parameters from the current worlds' global neutrino data sample and discuss in detail the status of the small parameters $\alpha \equiv \Delta m_{\mathrm{SOL}}^{2} / \Delta m_{\mathrm{ATM}}^{2}$ as well as $\sin ^{2} \theta_{13}$, which characterize the strength of $\mathrm{CP}$ violating effects in neutrino oscillations. We also update the degree of rejection of four-neutrino interpretations of the LSND evidence in view of the most recent developments.
\end{abstract}

PACS numbers: 26.65.+t, 13.15.+g, 14.60.Pq, 95.55.Vj 


\section{Introduction}

The discovery of neutrino masses by the combination of a variety of data from solar [1, 2, 3, 4, 5, 6, 7, 8, 9, 10], atmospheric [1], 12, 13], reactor 14, 15] and accelerator [16, 17] neutrino experiments was the major recent achievement in astroparticle, high energy, and nuclear physics, which culminates a heroic effort dating back to about four decades. This has now firmly established the incompleteness of the Standard Model of electroweak interactions, expected on theoretical grounds since long ago 18, 19, 20, 21, 22]. The determination of neutrino oscillation parameters is now a flourishing industry [23] which has finally entered the high precision age, with many experiments underway and a new generation coming. Apart from a careful understanding of solar and atmospheric neutrino fluxes, nuclear physics, neutrino cross sections and experimental response functions, the interpretation of the data relies heavily on the proper description of neutrino propagation properties both in the Sun and the Earth, including the so-called matter effects [24, 25].

The avalanche of data in a field where these have been traditionally so scarce, given the feebleness of neutrino interactions, has prompted a rush of phenomenological papers on the interpretation of neutrino data. A number of reviews are already in the market [26, 27, 28, 29]. Here we revisit the latest global analysis of neutrino oscillation parameters presented in Ref. 30] in view of the most recent solar [31] and atmospheric [32] neutrino flux calculations and of the new data presented at Neutrino 2004.

This paper is organized as follows. In Sec. 2 we briefly discuss the analysis of the atmospheric neutrino data including the state-of-the-art three-dimensional calculation of the atmospheric neutrino flux given in Ref. [32]. In order to obtain the allowed ranges for the oscillation parameters $\Delta m_{\mathrm{ATM}}^{2}$ and $\theta_{\mathrm{ATM}}$ we combine the atmospheric neutrino data from the Super-K experiment with the accelerator data from the K2K experiment, which provides the first independent confirmation of the oscillation evidence from atmospheric neutrinos. We give also a short description of robustness of the atmospheric data with respect to non-standard neutrino interactions in Sec. 2.4

In Sec. 3 we briefly describe the solar neutrino experiments and the KamLAND reactor neutrino experiment, and discuss the two-neutrino interpretation of the data described by $\Delta m_{\mathrm{SOL}}^{2}$ and $\theta_{\mathrm{SOL}}$. We comment on the implications of the recent update of the Standard Solar Model (SSM) neutrino fluxes from Ref. 31]. Furthermore, in Sec. 3.4 we discuss the robustness of the oscillation interpretation of solar neutrino data when going beyond the SSM framework or invoking non-standard neutrino properties. As examples we consider the effects of solar radiative-zone density fluctuations, convectivezone magnetic fields and the prospects of probing electromagnetic neutrino properties with current and future experiments.

After discussing the dominant oscillations in the two-flavour approximation in Secs. 2 and 3 we devote Sec. 4 to the global three-neutrino analysis of the data, combining all current neutrino oscillation data except LSND. In addition to presenting 
the best fit values and allowed ranges for the oscillation parameters, we update the status of the three-flavour parameters $\alpha \equiv \Delta m_{\mathrm{SOL}}^{2} / \Delta m_{\mathrm{ATM}}^{2}$ and $\theta_{13}$ in view of the new data reported at Neutrino 2004. In Sec. [5 we give an update of the status of attempts to account for the LSND data in terms of four-neutrino oscillations. Finally we conclude and summarize our results in Sec. 6. A detailed discussion of the recent KamLAND results is given in Appendix A

\section{Leading oscillations with $\Delta m_{\text {atm }}^{2}$}

\subsection{Atmospheric neutrino oscillations}

In 1998 the Super-K experiment obtained evidence for neutrino oscillations [1] from the observation of the zenith angle dependence of their $\mu$-like atmospheric neutrino data. This effect has been confirmed also by other atmospheric neutrino experiments, see e.g. Refs. 12, 13]. Recently, Super-K has reported a dip in the $L / E$ distribution of the atmospheric $\nu_{\mu}$ survival probability 33], which provides a clear signature for neutrino oscillations.

In our atmospheric neutrino analysis we include the most recent charged-current atmospheric neutrino data from Super-K [34], including the $e$-like and $\mu$-like data samples of sub- and multi-GeV contained events (each grouped into 10 bins in zenith angle), as well as the stopping (5 angular bins) and through-going (10 angular bins) upgoing muon data events. As previously, we do not use the information on $\nu_{\tau}$ appearance, multi-ring $\mu$ and neutral-current events since an efficient Monte-Carlo simulation of these data would require a more detailed knowledge of the Super-K experiment, and in particular of the way the neutral-current signal is extracted from the data. For details of our analysis see Refs. [35, 36] and references therein. With respect to our previous atmospheric neutrino analysis [30] we have now taken into account the new three-dimensional atmospheric neutrino fluxes given in Ref. 32]. Furthermore, we have updated our statistical analysis following closely Ref. 37], taking special care of systematical errors, like uncertainties in the neutrino fluxes and detection cross sections. Details on the $\chi^{2}$ analysis can also be found in Ref. [38]. With these updates our results are in excellent agreement with the ones of the Super-K collaboration [34].

In Fig. 1 we show the results of our analysis of atmospheric data in the framework of two-flavour $\nu_{\mu} \rightarrow \nu_{\tau}$ oscillations. The regions delimited by the hollow contours correspond to the allowed regions for the oscillation parameters $\sin ^{2} \theta_{\text {AтM }}$ and $\Delta m_{\text {АTM }}^{2}$. The current best fit point occurs at

$$
\left.\sin ^{2} \theta_{\text {ATM }}=0.50, \quad \Delta m_{\mathrm{ATM}}^{2}=2.0 \times 10^{-3} \mathrm{eV}^{2} \quad \text { (ATM data }\right) .
$$

The main difference to the results of Ref. [30] are the relatively lower values of $\Delta m_{\mathrm{ATM}}^{2}$ implied by the use of the new three-dimensional atmospheric neutrino fluxes reported in Ref. 32] instead of the one-dimensional Bartol fluxes [39] used previously. 


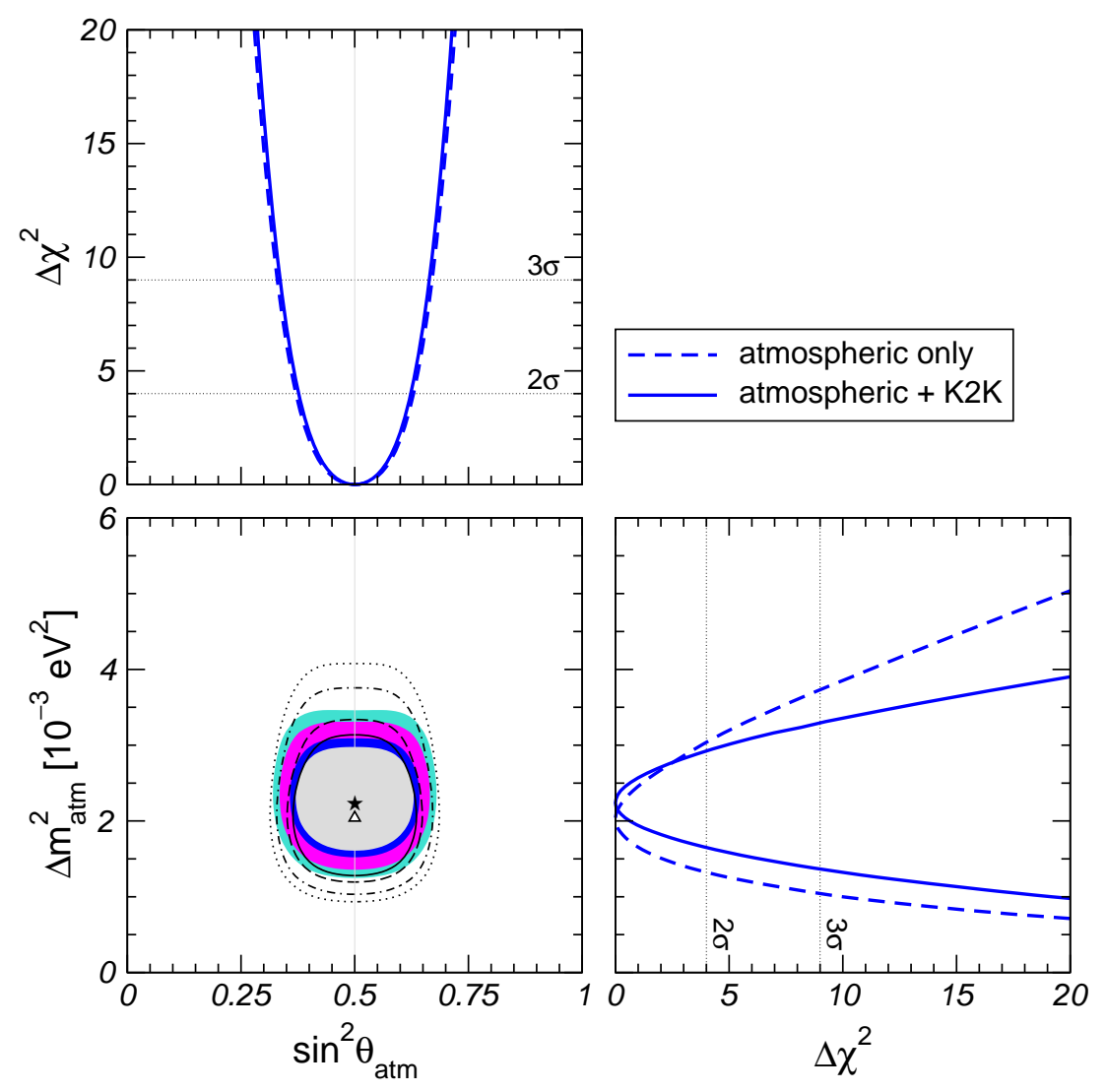

Figure 1. Allowed $\left(\sin ^{2} \theta_{\text {ATM }}, \Delta m_{\text {ATM }}^{2}\right)$ regions at $90 \%, 95 \%, 99 \%$, and $3 \sigma$ C.L. for 2 d.o.f. The regions delimited by the lines correspond to atmospheric data only, while for the colored regions also $\mathrm{K} 2 \mathrm{~K}$ data are added. The best fit point of atmospheric (atmospheric $+\mathrm{K} 2 \mathrm{~K}$ ) data is marked by a triangle (star). Also shown is the $\Delta \chi^{2}$ as a function of $\sin ^{2} \theta_{\mathrm{ATM}}$ and $\Delta m_{\mathrm{ATM}}^{2}$, minimized with respect to the undisplayed parameter.

\subsection{The K2K accelerator experiment}

The KEK to Kamioka (K2K) long-baseline neutrino oscillation experiment [16] probes the $\nu_{\mu}$ disappearance oscillation channel in the same region of $\Delta m^{2}$ as explored by atmospheric neutrinos. The neutrino beam is produced by a $12 \mathrm{GeV}$ proton beam from the KEK proton synchrotron, and consists of $98 \%$ muon neutrinos with a mean energy of $1.3 \mathrm{GeV}$. The beam is controlled by a near detector $300 \mathrm{~m}$ away from the proton target. Information on neutrino oscillations is obtained by the comparison of this near detector data with the $\nu_{\mu}$ content of the beam observed by the Super-Kamiokande detector at a distance of $250 \mathrm{~km}$.

The data sample called K2K-I 16] has been collected in the period from June 1999 to July $2001\left(4.8 \times 10^{19}\right.$ protons on target). K2K-I has observed 56 events in Super-K, whereas $80.1_{-5.4}^{+6.2}$ have been expected in the case of no oscillations. This gives a clear evidence for $\nu_{\mu}$ disappearance: the probability that the observed flux at Super- $\mathrm{K}$ is explained by a statistical fluctuation without neutrino oscillations is less than $1 \%$ [16]. Recently, at the Neutrino2004 conference new data from the K2K-II 
period have been presented [17]. K2K-II started in fall 2002, and the released data corresponds to $4.1 \times 10^{19}$ protons on target, comparable to the K2K-I sample. From the combined analysis of K2K-I and K2K-II 108 events have been observed in Super-K, whereas $150.9_{-10.0}^{+11.6}$ have been expected for no oscillations. Out of the 108 events 56 are so-called single-ring muon events. This data sample contains mainly muon events from the quasi-elastic scattering $\nu_{\mu}+p \rightarrow \mu+n$, and the reconstructed energy is closely related to the true neutrino energy. Hence these data can be used for a spectral analysis. Using the Kolmogorov-Smirnov test the K2K collaboration finds that the observed spectrum is consistent with the spectrum expected for no oscillation only at a probability of $0.11 \%$, whereas the spectrum predicted by the best fit oscillation parameters has a probability of $52 \%$ [17].

In our re-analysis of $\mathrm{K} 2 \mathrm{~K}$ data we use the energy spectrum of the 56 single-ring muon events from K2K-I + K2K-II ¥. Similar as in Ref. [38] we use a phenomenological parameterization for the spectrum expected for no oscillation. Adopting reasonable assumptions on the energy resolution function and on systematical errors, we fit the data divided into 15 bins in reconstructed neutrino energy, as given in Ref. 17]. The allowed regions for the oscillation parameters from $\mathrm{K} 2 \mathrm{~K}$ data are shown in Fig. 2 in comparison to the ones from atmospheric neutrino data. This figure illustrates that the neutrino mass-squared difference indicated by the $\nu_{\mu}$ disappearance observed in $\mathrm{K} 2 \mathrm{~K}$ is in perfect agreement with atmospheric neutrino oscillations. Hence, K2K data provide the first confirmation of oscillations with $\Delta m_{\text {ATM }}^{2}$ from a man-made neutrino source. $\mathrm{K} 2 \mathrm{~K}$ gives a rather weak constraint on the mixing angle due to low statistics in the current data sample.

\subsection{Atmospheric and K2K combined}

In Fig. 11 the allowed regions in the $\left(\sin ^{2} \theta_{\text {ATM }}, \Delta m_{\text {ATM }}^{2}\right)$ plane from the combined analysis of $\mathrm{K} 2 \mathrm{~K}$ and Super-K atmospheric neutrino data are shown as shaded regions. As expected from Fig. 2 we find that, apart from providing an independent confirmation, $\mathrm{K} 2 \mathrm{~K}$ data start already to constrain the allowed region of $\Delta m_{\mathrm{ATM}}^{2}$, whereas the determination of the mixing angle is completely dominated by atmospheric data. From the projections of the $\chi^{2}$ onto the $\Delta m_{\text {ATM }}^{2}$ and $\sin ^{2} \theta_{\text {Aтм }}$ axes shown in Fig. 1 we find the best fit point

$$
\sin ^{2} \theta_{\mathrm{ATM}}=0.5, \quad \Delta m_{\mathrm{ATM}}^{2}=2.2 \times 10^{-3} \mathrm{eV}^{2} \quad(\mathrm{ATM}+\mathrm{K} 2 \mathrm{~K} \text { data })
$$

with the corresponding allowed ranges at $3 \sigma(5 \sigma)$ for 1 d.o.f.:

$$
\begin{aligned}
& 0.34(0.27) \leq \sin ^{2} \theta_{\text {ATM }} \leq 0.66(0.73) \\
& 1.4(0.85) \times 10^{-3} \mathrm{eV}^{2} \leq \Delta m_{\mathrm{ATM}}^{2} \leq 3.3(4.2) \times 10^{-3} \mathrm{eV}^{2}
\end{aligned}
$$

Note that despite the downward shift of the atmospheric mass-splitting implied by the new neutrino fluxes from Ref. [32] our new quoted value for $\Delta m_{\mathrm{ATM}}^{2}$ in Eq. (21) is $\ddagger$ We cannot use the full K2K data sample of 108 events, since not enough information is available to analyze these data outside the K2K collaboration. 


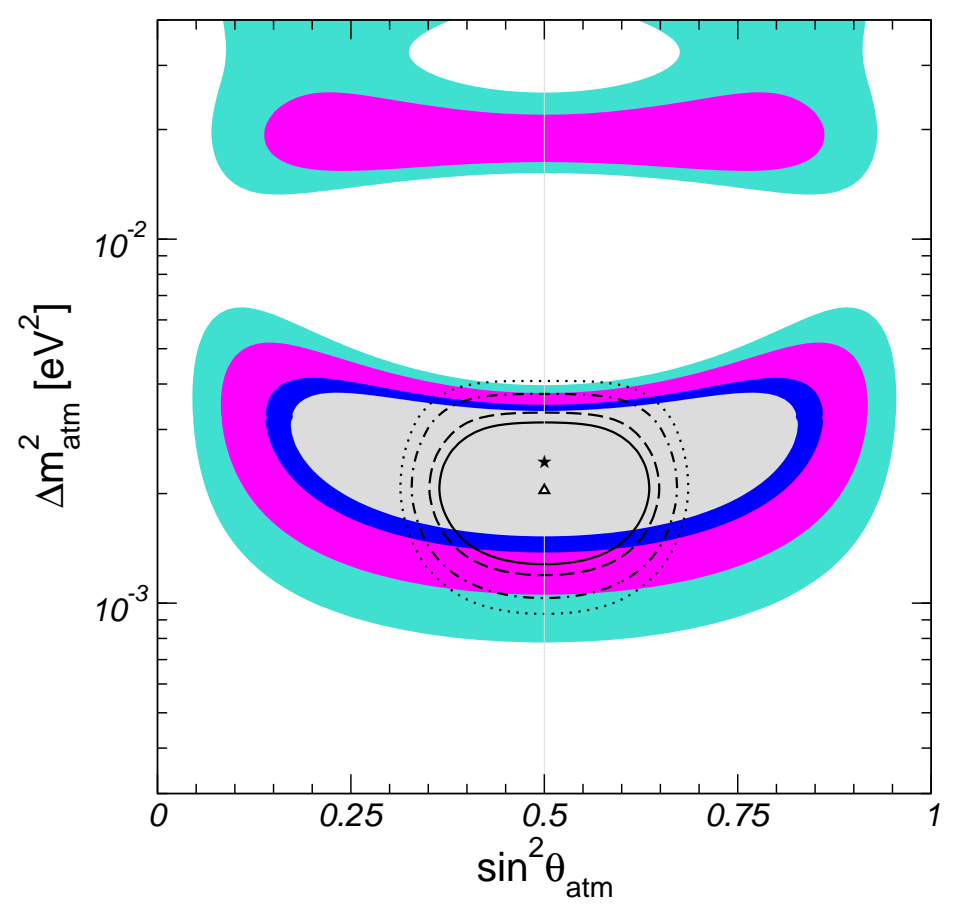

Figure 2. Allowed K2K regions in the $\left(\sin ^{2} \theta_{\text {ATM }}, \Delta m_{\text {AтM }}^{2}\right)$ plane at $90 \%, 95 \%, 99 \%$, and $3 \sigma$ C.L. for 2 d.o.f. The hollow lines delimit the region determined from the atmospheric data only. The star (triangle) corresponds to the K2K (atmospheric) best fit point.

statistically compatible both with our previous result 30. and the value obtained by the new Super-K $L / E$ analysis [33]. Let us remark that the K2K constraint on $\Delta m_{\text {Атм }}^{2}$ from below is important for future long-baseline experiments, since the performance of such experiments is drastically affected if $\Delta m_{\mathrm{ATM}}^{2}$ were in the lower part of the $3 \sigma$ range allowed by atmospheric data (see e.g. Ref. [40]).

\subsection{Robustness of oscillation interpretation: atmospheric neutrinos}

We now turn to the issue of the robustness of the oscillation interpretation of the atmospheric neutrino data. Non-standard physics may in principle affect atmospheric neutrino fluxes, as well as neutrino propagation and detection cross sections 26]. Apart from the issue of their theoretical viability [41] neutrino decays have been considered since long ago [42]. Non-standard interactions arising from the $\mathrm{SU}(2) \otimes \mathrm{U}(1)$ charged and neutral currents [20] or from new particles [43, 44, 45, 46, 47, 48, 49], as well as quantum mechanical decoherence [38] might also affect atmospheric neutrino results. Although strongly rejected by recent atmospheric data as the dominant mechanisms [33, 38, 50], non-standard phenomena might still be present at a subleading level in addition to oscillations and, to this extent, have some impact on the determination of the oscillation parameters.

In the following we illustrate the stability of the measurement of $\Delta m_{\mathrm{ATM}}^{2}$ and $\sin ^{2} \theta_{\text {ATM }}$ by assuming the presence of non-standard interactions of the neutrinos with 


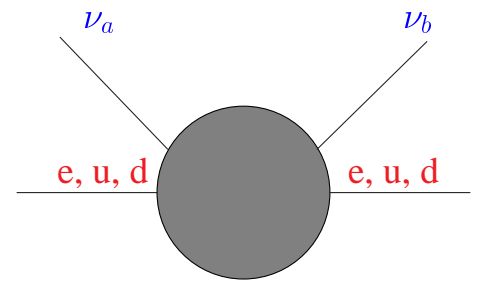

Figure 3. Effective flavour-changing NSI operator.

earth matter. New neutrino interactions beyond the Standard Model are a natural feature in most neutrino mass models [51] and can be of two types: flavour-changing (FC) and non-universal (NU). These interactions (called NSI for short) may be schematically represented as effective dimension- 6 terms of the type $\varepsilon G_{F}$, as illustrated in Fig. 3, where $\varepsilon$ specifies their sub-weak strength. Such interactions may arise from a nontrivial structure of charged and neutral current weak interactions characterized by a non-unitary lepton mixing matrix [20]. These gauge-induced NSI may lead to flavour and $\mathrm{CP}$ violation, even with massless or degenerate neutrinos [45, 47, 48, 49]. Alternatively, such non-standard neutrino interactions may also arise in models where neutrino masses are "calculable" from radiative corrections [43, 44] and in some supersymmetric models with broken R parity [52]. Finally, in supersymmetric unified models, the strength of non-standard neutrino interactions may be a calculable renormalization effect [46].

The impact of non-standard neutrino interactions on atmospheric neutrinos was considered in Ref. [50] treating the NSI strengths as free phenomenological parameters. This analysis takes into account both the effect of $\nu_{\mu} \rightarrow \nu_{\tau}$ oscillations (OSC) as well as the existence of non-standard neutrino-matter interactions (NSI) in this channel. In addition to the standard term in the Hamiltonian describing oscillations an term $H_{\mathrm{NSI}}$ is introduced, accounting for an effective potential induced by the NSI with earth matter:

$$
H_{\mathrm{NSI}}= \pm \sqrt{2} G_{F} N_{f}\left(\begin{array}{cc}
0 & \varepsilon \\
\varepsilon & \varepsilon^{\prime}
\end{array}\right) .
$$

Here $+(-)$ holds for neutrinos (anti-neutrinos) and $\varepsilon$ and $\varepsilon^{\prime}$ parameterize the NSI: $\sqrt{2} G_{F} N_{f} \varepsilon$ is the forward scattering amplitude for the FC process $\nu_{\mu}+f \rightarrow \nu_{\tau}+f$ and $\sqrt{2} G_{F} N_{f} \varepsilon^{\prime}$ represents the difference between $\nu_{\mu}+f$ and $\nu_{\tau}+f$ elastic forward scattering. The quantity $N_{f}$ is the number density of the fermion $f$ along the neutrino path. For definiteness we take for $f$ the down-type quark.

In Fig. 4 we show the results of a fit to atmospheric neutrino data for the four parameters $\Delta m_{\mathrm{ATM}}^{2}, \sin ^{2} 2 \theta_{\mathrm{ATM}}, \varepsilon$, and $\varepsilon^{\prime} \S$. In the left panels the pure oscillation case $\left(\varepsilon=\varepsilon^{\prime}=0\right)$ is compared to the case where also some NSI are allowed. We find that the $\chi^{2}$ improves slightly (2.4 units) in the presence of NSI, but the determination of the oscillation parameters is practically unaffected. This is an important result, since it shows that the allowed ranges derived for $\Delta m_{\text {ATM }}^{2}$ and $\sin ^{2} 2 \theta_{\text {ATM }}$ are rather stable $\S$ Here we assume that the parameter $\varepsilon$ is real; for the more general case of complex $\varepsilon$, see Ref. [53]. 

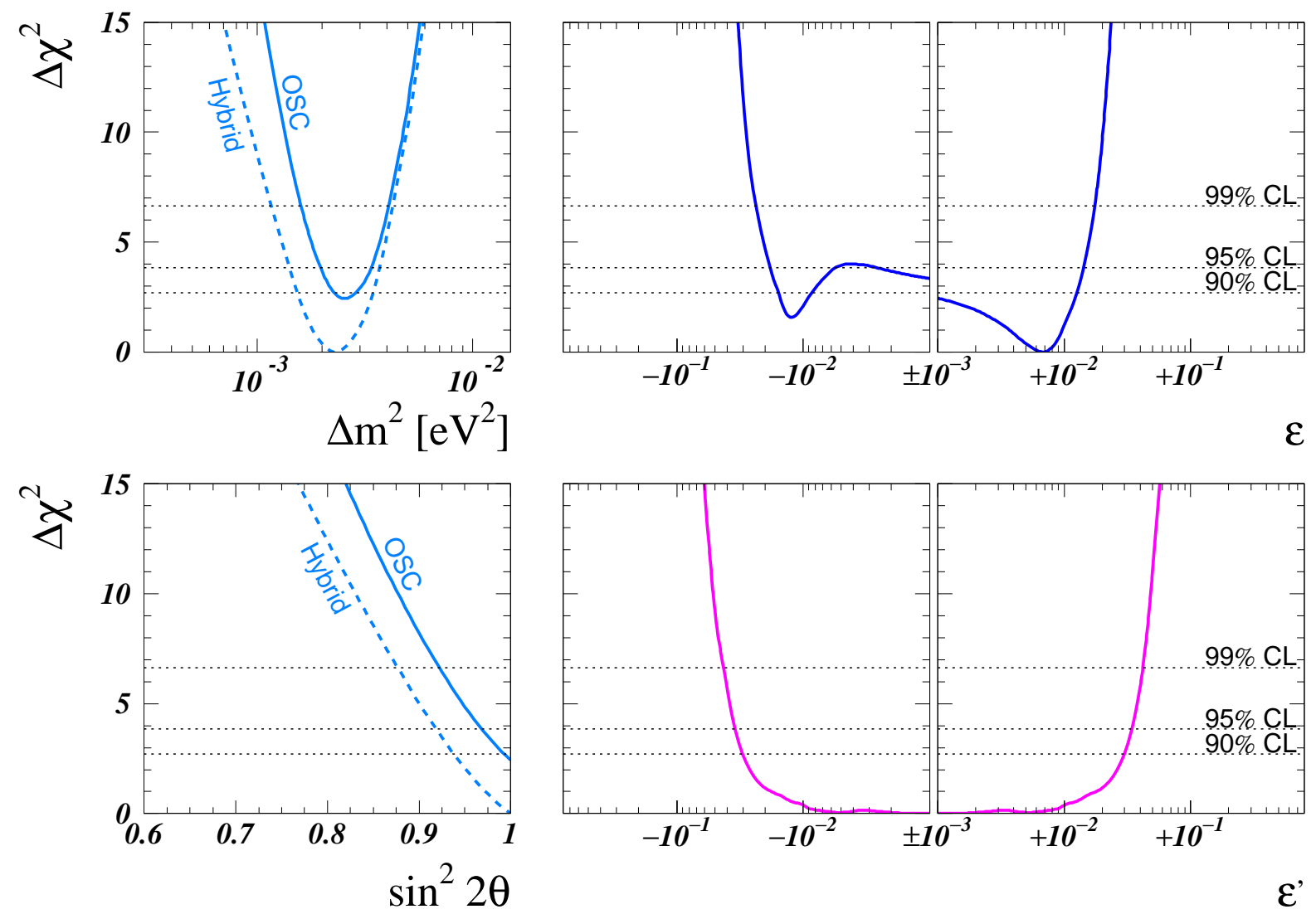

Figure 4. Atmospheric neutrino oscillation parameters from [50] fitted in the presence of non-standard interactions using the Bartol flux [39]. We show the behaviour of the $\chi^{2}$ as a function of the four parameters $\Delta m_{\text {ATM }}^{2}$ (upper left), $\sin ^{2} 2 \theta_{\text {АTM }}$ (lower left), the FC parameter $\varepsilon$ (upper right), and the NU parameter $\varepsilon^{\prime}$ (lower right). In each panel the $\chi^{2}$ is minimized with respect to the three undisplayed parameters. In the left panels we show also the pure oscillation case (OSC), with $\varepsilon$ and $\varepsilon^{\prime}$ set to zero.

with respect to non-standard physics $\|$. In turn, the high preference of the data for oscillations allows to set strong bounds on NSI. From the right panels of Fig. 4 we deduce the bounds at $3 \sigma$

$$
-0.03 \leq \varepsilon \leq 0.02, \quad\left|\varepsilon^{\prime}\right| \leq 0.05 .
$$

Before closing we stress that, apart from its intrinsic theoretical importance, the study of non-standard neutrino interactions has an astrophysical interest, as these can affect the propagation of neutrinos in a variety of astrophysical environments, such as supernovae [54, 55] and pulsars [56]. They could lead to "deep-inside" neutrino conversions, in addition to those expected from conventional neutrino oscillations.

|| Note that the analysis of Ref. [50] is based on the neutrino fluxes of Ref. 39], and in addition to the Super-K data described in Sec. 2.1 also the up-going muon data from the MACRO experiment 12] is

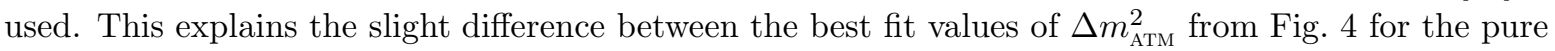
oscillation case and Eq. (11). 


\section{Leading oscillations with $\Delta m_{\mathrm{sol}}^{2}$}

\subsection{Solar neutrino oscillations}

In this section we consider oscillations of solar neutrinos in the two-flavour framework. A detailed discussion of experimental and theoretical aspects of solar neutrino physics can be found in other contributions to this volume [57, 58]. Therefore, we focus in the following on the determination of the oscillation parameters from the fit to solar neutrino data. In our analysis we take into account the rates of the chlorine experiment at the Homestake mine [1, 2] $(2.56 \pm 0.16 \pm 0.16 \mathrm{SNU})$, the most up-to-date results 59] of the gallium experiments SAGE [3, 60] (66.9 $\left.{ }_{-3.8}^{+3.9}{ }_{-3.2}^{+3.6} \mathrm{SNU}\right)$ and GALLEX/GNO [4, 5 , 6 ] $(69.3 \pm 4.1 \pm 3.6 \mathrm{SNU})$, as well as the 1496-day Super-K data sample [7] in the form of 44 bins ( 8 energy bins, 6 of which are further divided into 7 zenith angle bins). From the SNO experiment we include the most recent data from the salt phase [10] in the form of the neutral current (NC), charged current (CC) and elastic scattering (ES) fluxes, as well as the 2002 spectral day/night data [8, 9] (17 energy bins for each day and night period).

The analysis methods used here are similar to the ones described in Refs. [30, 36] and references therein, including the use of the so-called pull approach for the $\chi^{2}$ calculation, as described in Ref. [61]. In this method all systematic uncertainties are included by introducing new parameters in the fit and adding a penalty function to the $\chi^{2}$. For example, for each of the eight solar neutrino fluxes a parameter is introduced, and the predictions from the Standard Solar Model including the correlated errors are taken into account by means of a penalty function. The method described in Ref. 61] is extended in two respects. First, it is generalized to the case of correlated statistical errors [62] as necessary to treat the SNO-salt data. Second, we do not consider the $\chi^{2}$ only up to first order in the pulls, but instead each pull parameter is treated exactly to all orders. This is particularly interesting in the case of the solar ${ }^{8} \mathrm{~B}$ flux. In our approach it is possible to include the SSM prediction for this flux as well as the SNO NC measurement on the same footing, without pre-selecting a particular value, as implied by expanding around the predicted value. In this way the fit itself can choose the best compromise between the SNO NC data and the SSM prediction.

In Fig. 5] we compare the allowed regions for the oscillation parameters using the solar neutrino fluxes given in the BP00 SSM [63] and the recent update (BP04) presented in Ref. [31]. One finds that the change in the flux predictions has a negligible impact on the allowed regions. This illustrates that thanks to the good experimental accuracy the determination of the oscillation parameters is rather robust with respect to changes in the SSM. The current best fit values for solar neutrino oscillation parameters are

$$
\sin ^{2} \theta_{\mathrm{SOL}}=0.29, \quad \Delta m_{\mathrm{SOL}}^{2}=6.0 \times 10^{-5} \mathrm{eV}^{2} \quad \text { (solar data, BP04).(6) }
$$

Also the rejection against maximal solar mixing is $5.6 \sigma$, the same as found previously 30 ] using the BP00 solar model. This is the significance at which bi-maximal models of neutrino mass, such as the CP conserving version of the neutrino unification model 


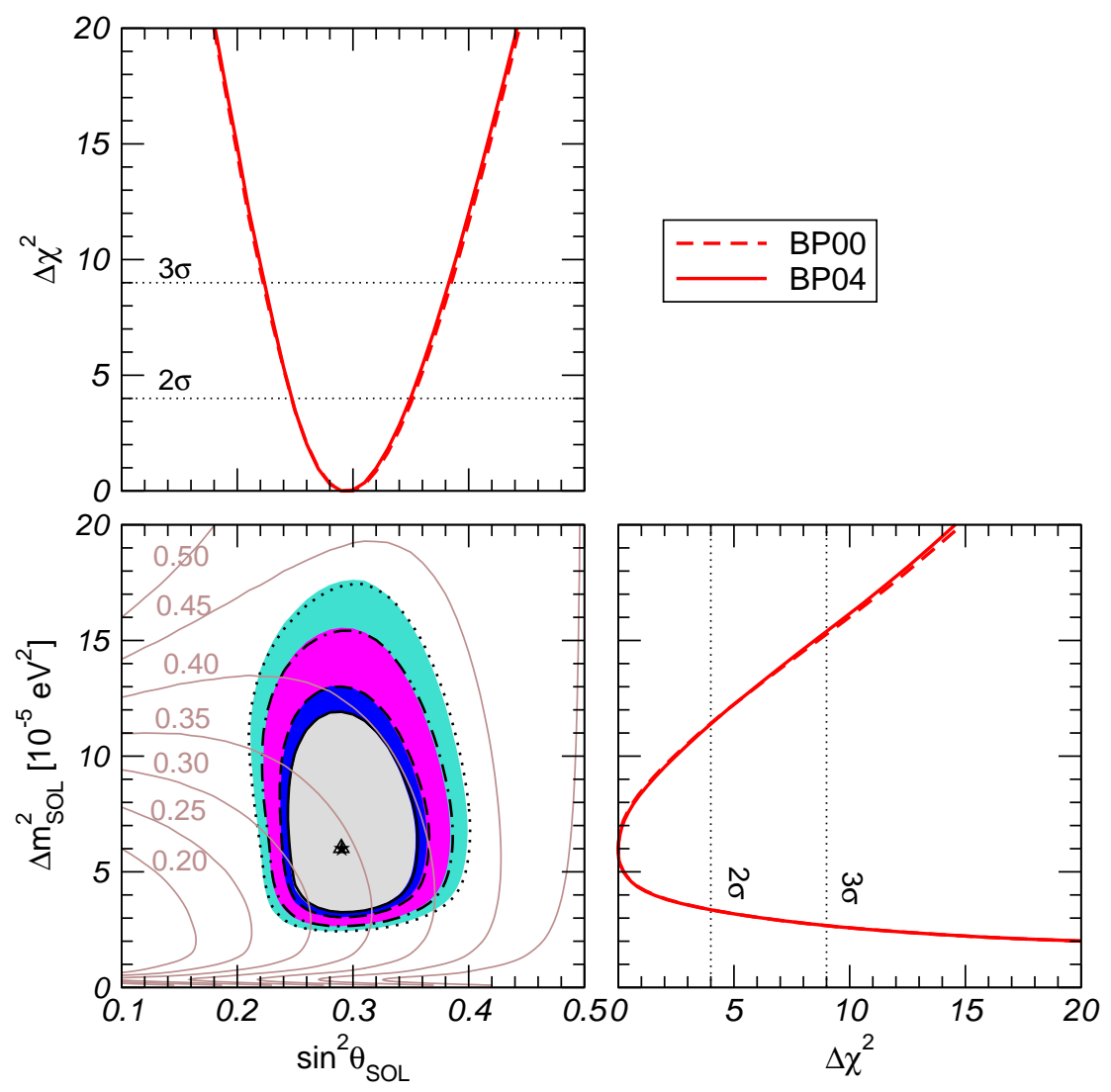

Figure 5. Allowed regions from all solar neutrino data at 90\%, 95\%, 99\%, and $3 \sigma$ C.L. for 2 d.o.f. in the plane of $\sin ^{2} \theta_{\mathrm{SOL}}$ and $\Delta m_{\mathrm{SOL}}^{2}$. The regions delimited by the curves correspond to the BP00 SSM [63], whereas for the colored regions the BP04 SSM 31] has been used. Also shown is $\Delta \chi^{2}$ as a function of $\sin ^{2} \theta_{\mathrm{SOL}}$ and $\Delta m_{\mathrm{SOL}}^{2}$, minimized with respect to the undisplayed parameter. The labeled contours denote constant $\mathrm{CC} / \mathrm{NC}$ ratio in the $\mathrm{SNO}$ experiment.

given in Ref. [64], are ruled out. Despite the fact that the BP04 model does not imply any significant change in the solar neutrino parameters, we will use it in our subsequent discussion of solar neutrino results.

\subsection{The KamLAND reactor neutrino experiment}

The KamLAND experiment [14, 15] is a reactor neutrino experiment with its detector located at the Kamiokande site. Most of the $\bar{\nu}_{e}$ flux incident at KamLAND comes from nuclear plants at distances of $80-350 \mathrm{~km}$ from the detector, making the average baseline of about 180 kilometers, long enough to provide a sensitive probe of the LMA solution of the solar neutrino problem. The KamLAND collaboration has for the first time measured the disappearance of neutrinos traveling to a detector from a power reactor. They observe a strong evidence for the disappearance of neutrinos during their flight over such distances, giving the first terrestrial confirmation of the solar neutrino anomaly and also establishing the oscillation hypothesis with man-produced neutrinos.

In KamLAND the reactor anti-neutrinos are observed by the process $\bar{\nu}_{e}+p \rightarrow$ 
$e^{+}+n$, where the delayed coincidence of the prompt energy from the positron and a characteristic gamma from the neutron capture allows an efficient reduction of backgrounds. The neutrino energy is related to the prompt energy by $E_{\nu}=E_{\mathrm{pr}}+\Delta-m_{e}$, where $\Delta$ is the neutron-proton mass difference and $m_{e}$ is the positron mass. In the lower part of the energy spectrum there is a relevant contribution from geo-neutrino events to the signal (see, e.g., Refs. 65, 66]). To avoid large uncertainties associated with the geo-neutrino flux an energy cut at $2.6 \mathrm{MeV}$ prompt energy is applied for the oscillation analysis.

First results from KamLAND were published in Ref. 14]. In the period from March to October 2002 data corresponding to a 162 ton-year exposure have been collected, and after all cuts 54 anti-neutrino events remained in the final sample. This number has to be compared with $86.8 \pm 5.6$ reactor neutrino events predicted for no oscillations and $0.95 \pm 0.99$ background events, which gives a probability that the KamLAND result is consistent with the no-disappearance hypothesis of less than $0.05 \%$ 14]. Recently, new results have been presented by KamLAND [15]. With a somewhat larger fiducial volume of the detector an exposure corresponding to 766.3 ton-year has been obtained between March 2002 and January 2004 (including a reanalysis of the 2002 data from Ref. 14]). A total of 258 events has been observed, in comparison to the expectation of $356.2 \pm 23.7$ reactor neutrino events in the case of no disappearance and $7.5 \pm 1.3$ background events. This leads to a confidence level of $99.995 \%$ for $\bar{\nu}_{e}$ disappearance, and the averaged survival probability is $0.686 \pm 0.044$ (stat) \pm 0.045 (syst). Moreover evidence for spectral distortion consistent with oscillations is obtained [15].

It was shown in Ref. 67] in relation with the first KamLAND data that most information on the neutrino oscillation parameters $\sin ^{2} \theta_{\mathrm{SOL}}$ and $\Delta m_{\mathrm{SOL}}^{2}$ can be extracted from the data by using an event-based likelihood analysis. This requires the knowledge of the prompt energy of each observed event. Unlike to the first data sample it is not possible to recover this information for the latest KamLAND data sample from publicly available material. Therefore only $\chi^{2}$-analyses based on binned data can be performed outside the KamLAND collaboration. Details of our KamLAND simulation based on 2002 data can be found in Refs. 67, 68], a discussion of our updated analysis method for the current data sample is given in Appendix A Here we briefly summarize the main features: Instead of the traditional bins of equal size in $E_{\mathrm{pr}}$ we use the data binned equally in $1 / E_{\mathrm{pr}}$, which can be obtained from Ref. [15], and which allows to extract more relevant information on the oscillation parameters. We adopt the improved anti-neutrino flux parameterization from Ref. 69], and include various systematic errors associated to the neutrino fluxes, reactor fuel composition and individual reactor powers. Furthermore, we include matter effects, a careful treatment of backgrounds and information on the average contribution to the total reactor neutrino signal as a function of the distance to the detector.

The KamLAND allowed regions for $\sin ^{2} \theta_{\mathrm{SOL}}$ and $\Delta m_{\mathrm{SOL}}^{2}$ are shown in Fig. [6] in comparison to the regions from solar data. One observes beautiful agreement between KamLAND data and the region implied by the LMA solution to the solar neutrino 

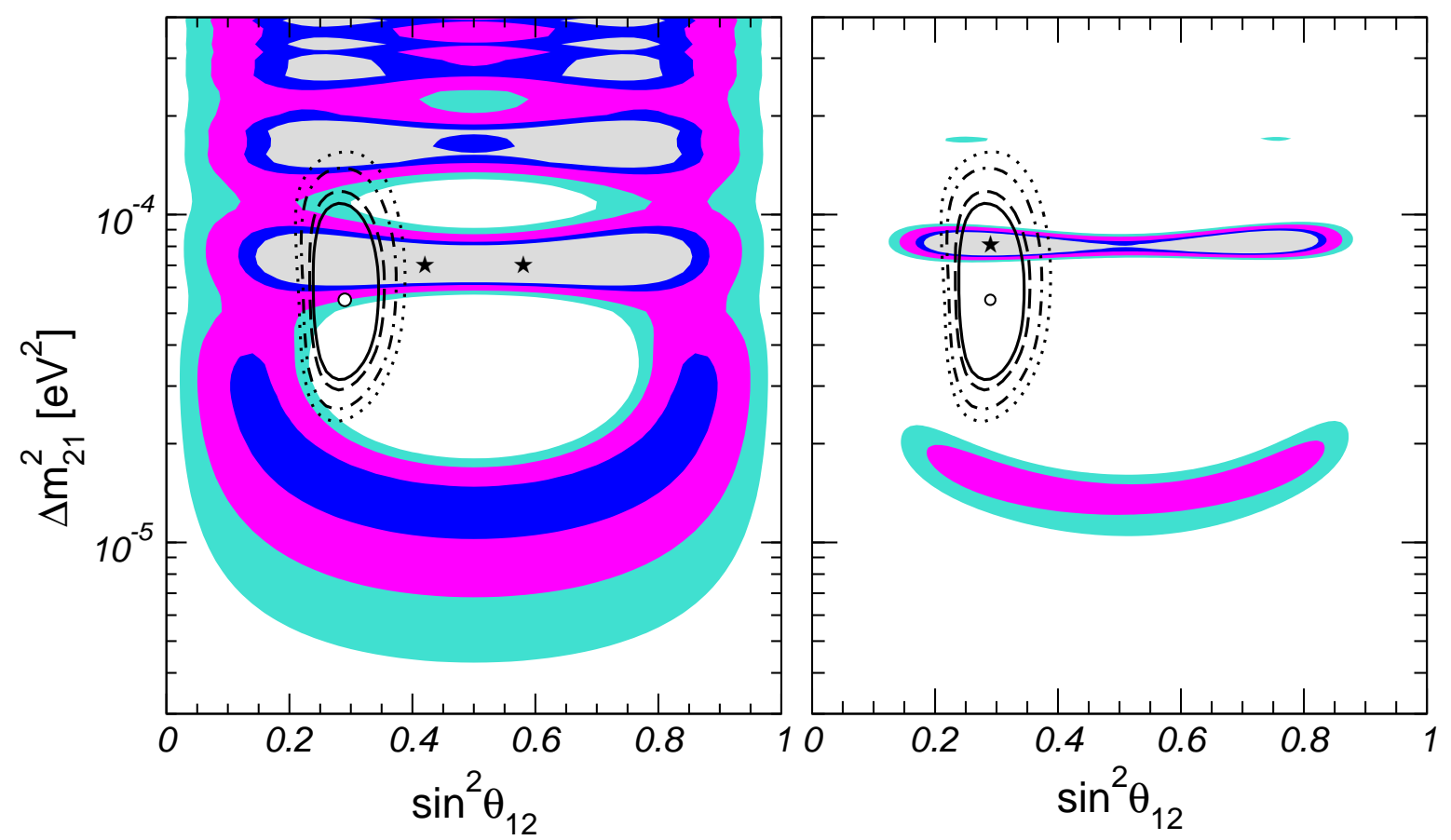

Figure 6. Allowed regions from KamLAND data at 90\%, 95\%, 99\%, and $3 \sigma$ C.L. for 2 d.o.f. for the 2002 data sample [14] (left panel) and the latest 766.3 ton-year data sample 15] (right panel). The regions delimited by the lines correspond to solar data. The KamLAND best fit points are marked with a star, the solar best fit point with a dot.

problem, which in this way has been singled out as the only viable one. Before this experiment we had a very complex pattern of alternative oscillation solutions like LOW, SMA, or VAC, see e.g., Refs. [35, 36]. All of these are completely ruled out by the KamLAND data. From this point of view the KamLAND experiment has played a fundamental role in the resolution of the solar neutrino problem.

Comparing the left and right panel of Fig. 6] one can appreciate the improvement implied by the recent KamLAND data. The allowed region is drastically reduced, and in particular the mass-squared difference is very well determined by recent data. We find the best fit point

$$
\begin{aligned}
& \Delta m_{\mathrm{SOL}}^{2}=8.1_{-0.3}^{+0.4} \times 10^{-5} \mathrm{eV}^{2}(1 \sigma) \quad \text { (KamLAND data). } \\
& \sin ^{2} \theta_{\mathrm{SOL}}=0.29
\end{aligned}
$$

The solution at low values of $\Delta m_{\mathrm{SOL}}^{2}$ is present at the $99 \%$ C.L., with the local minimum at $\Delta m_{\mathrm{SOL}}^{2}=1.6 \times 10^{-5} \mathrm{eV}^{2}$ and $\sin ^{2} \theta_{\mathrm{SOL}}=0.31$ and $\Delta \chi^{2}=7.5$ relative to the best fit point. The so-called high-LMA solution is present only marginally at the $3 \sigma$ level at $\Delta m_{\mathrm{SOL}}^{2}=1.7 \times 10^{-4} \mathrm{eV}^{2}, \sin ^{2} \theta_{\mathrm{SOL}}=0.25$ and $\Delta \chi^{2}=11.3$. Although not statistically significant, we note that the small matter effect favours slightly values of $\sin ^{2} \theta_{\text {SOL }}<0.5$ over the "mirror solution" with $\sin ^{2} \theta_{\mathrm{SOL}}>0.5$. The high values of $\Delta m_{\mathrm{SOL}}^{2} \gtrsim 3 \times 10^{-4} \mathrm{eV}^{2}$ in the averaging regime, which have been allowed by first KamLAND data 14] at $90 \%$ C.L. are now ruled out at more than $3 \sigma$. This indicates that the spectral distortion 


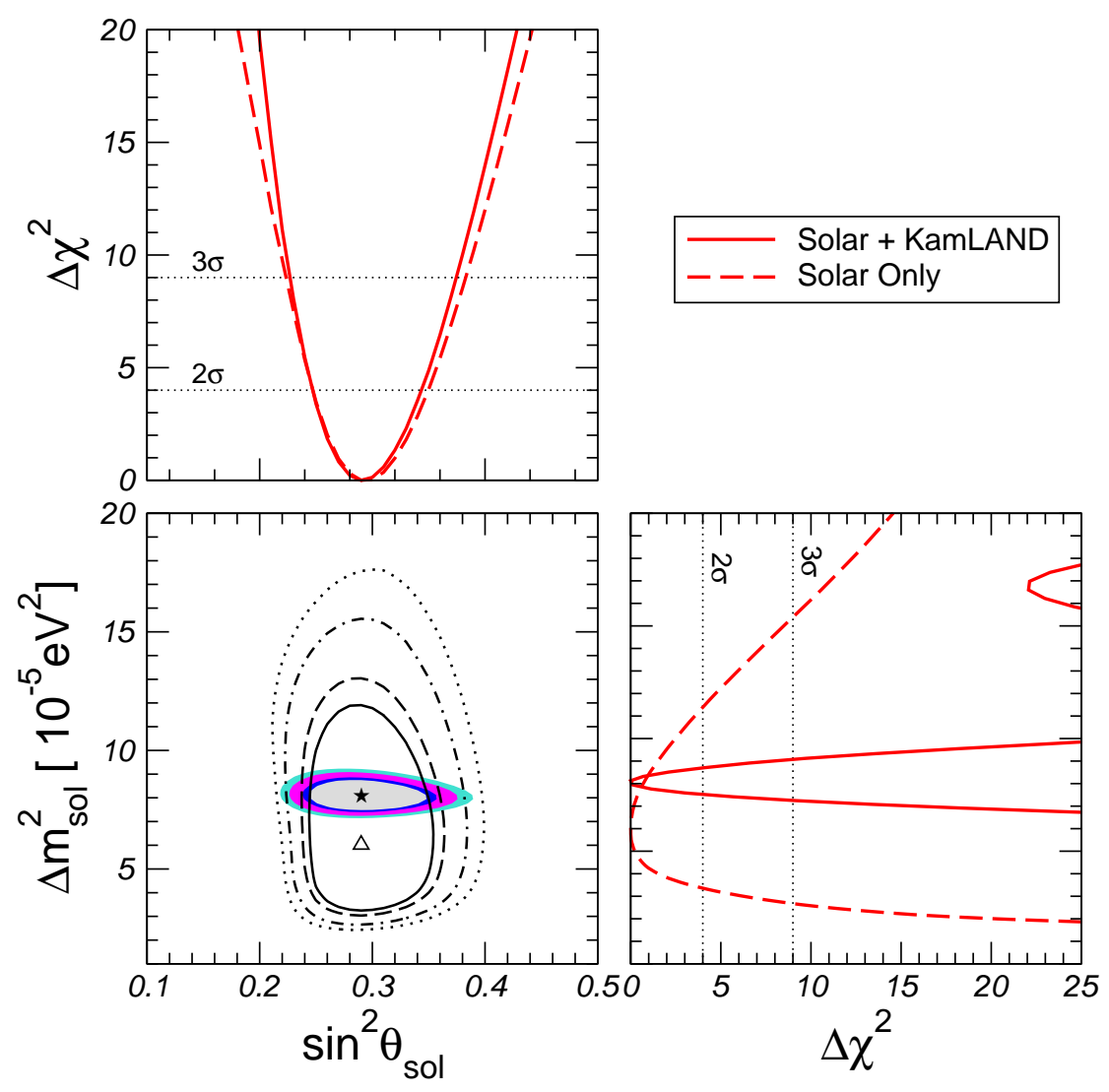

Figure 7. Allowed regions from combined solar and KamLAND data at 90\%, 95\%, $99 \%$, and $3 \sigma$ C.L. for 2 d.o.f.. The regions shown with lines correspond to solar data only, from Fig. 圃.

associated to neutrino oscillations has been observed [15] (see also Appendix A for further discussion).

\subsection{Solar and KamLAND combined}

Under the fundamental assumption of CPT invariance we can directly compare the information obtained from solar neutrino experiments and the KamLAND reactor experiment. In Fig. 7 we show the allowed regions from the combined solar and KamLAND data. The current best fit point of the global analysis occurs at

$$
\sin ^{2} \theta_{\mathrm{SOL}}=0.29, \quad \Delta m_{\mathrm{SOL}}^{2}=8.1 \times 10^{-5} \mathrm{eV}^{2} \quad(\text { Solar+KamLAND data).(8) }
$$

From the projections of the $\chi^{2}$ onto the $\Delta m_{\mathrm{SOL}}^{2}$ and $\sin ^{2} \theta_{\mathrm{SOL}}$ axes also shown in Fig. 7 we find the following allowed ranges at $3 \sigma(5 \sigma)$ for 1 d.o.f.:

$$
\begin{aligned}
& 0.23(0.19) \leq \sin ^{2} \theta_{\mathrm{SOL}} \leq 0.37(0.45) \\
& 7.3(6.7) \times 10^{-5} \mathrm{eV}^{2} \leq \Delta m_{\mathrm{SOL}}^{2} \leq 9.1(9.9) \times 10^{-5} \mathrm{eV}^{2}
\end{aligned}
$$

As expected from Fig. [ the determination of the mixing angle is completely dominated by the solar neutrino data, whereas KamLAND significantly reduces the allowed range for $\Delta m_{\mathrm{SOL}}^{2}$. Also the so-called high-LMA region, which previously was present around 


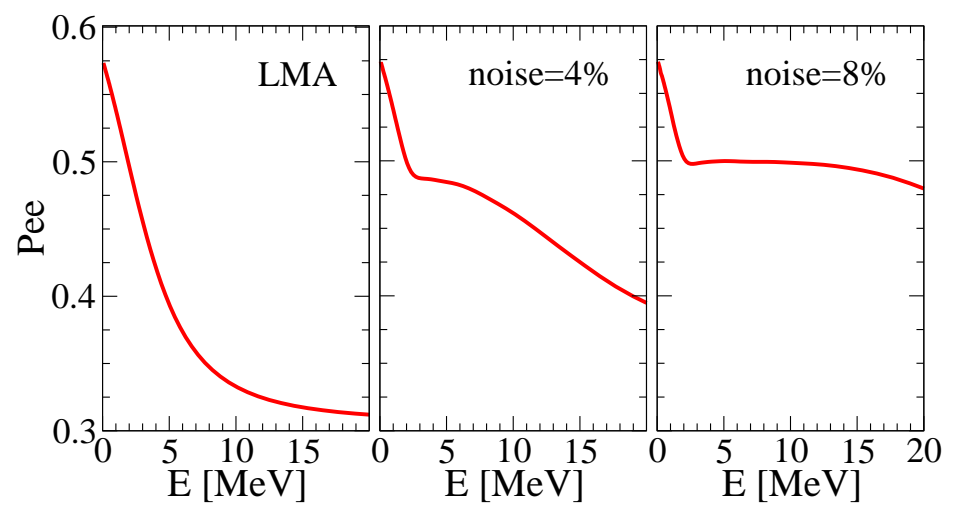

Figure 8. Effect of random matter density fluctuations with a correlation length of $L_{0}=100 \mathrm{~km}$ on the electron-neutrino survival probability for LMA oscillations.

$\Delta m_{\mathrm{SOL}}^{2} \simeq 1.4 \times 10^{-4} \mathrm{eV}^{2}$ at $3 \sigma$ (see e.g., Ref. [30]) is now ruled out with a $\Delta \chi^{2}=22.1$ with respect to the global minimum, which corresponds to an exclusion at about $4.3 \sigma$ for 2 d.o.f.. Notice also that the day/night data are treated as previously [30, 36]. An improved analysis of the day/night asymmetry data along the lines followed by the Super-K collaboration in Ref. [70] would lead to an even more pronounced rejection of the high $\Delta m_{\mathrm{SOL}}^{2}$ region. Currently not enough information is available to reproduce this result outside the Super-K collaboration. Finally we note that the use of the BP04 fluxes has not changed significantly the allowed regions.

\subsection{Robustness of oscillation interpretation: solar neutrinos}

The oscillation interpretation of solar neutrino data depends both on astrophysical input (the model of the Sun) as well as on the physics characterizing both the propagation as well as neutrino interaction properties. Either may differ from the Standard Solar Model and Standard Electroweak Model expectations. How robust is the oscillation interpretation of solar neutrino data in view of this?

3.4.1. Beyond the Standard Solar Model In the following we will briefly discuss consequences of departures from the Standard Solar Model. The effect of varying solar neutrino fluxes has been widely discussed, thus, as a case study we consider the possibility of solar density fluctuations. Even though this possibility was suggested in a number of papers [71, 72, 73] it has been traditionally neglected for several reasons. First, helioseismic measurements constrain deviations of solar properties from Standard Solar Model predictions at better than the percent level. Second, preliminary studies of the implications for neutrino oscillations of radiative-zone helioseismic waves 73] indicated that they were unlikely to have observable effects. Third, no other known sources of fluctuations seemed to have the properties required to influence neutrino oscillations.

Recently all of these points have been re-examined, with the result that the presence of solar fluctuations appears more likely than previously thought. First, direct 


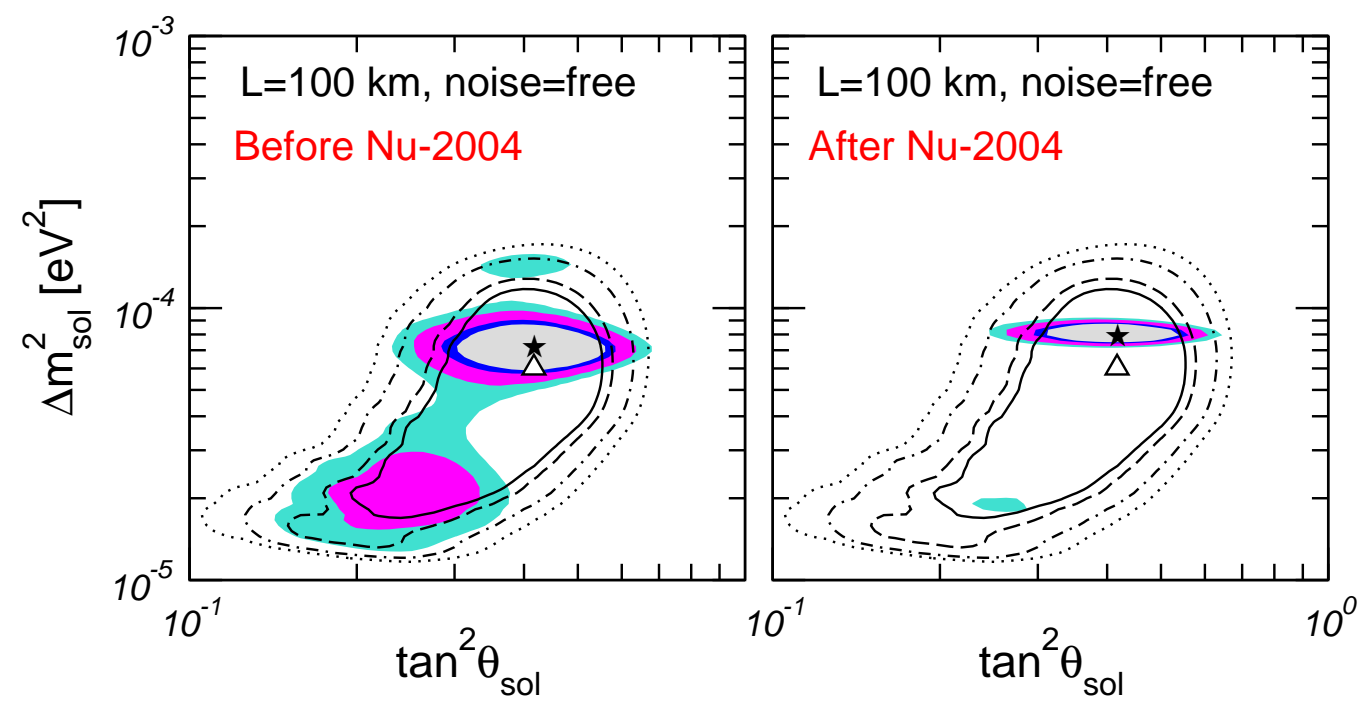

Figure 9. Solar neutrino oscillation parameters with an arbitrary noise amplitude and a correlation length $L_{0}=100 \mathrm{~km}$ from combining solar data with 2002 KamLAND data (left panel) and 2004 KamLAND data (right panel). The contour lines refer to solar data only.

helioseismic bounds turn out to be insensitive to fluctuations whose size is as small as those to which neutrinos are sensitive [74, 75] typically several hundreds of $\mathrm{km}$. Second, recent studies have shown how such solar density fluctuations can arise near the solar equatorial plane in the presence of magnetic fields deep within the solar radiative zone due to a resonance between Alfvén waves and helioseismic $g$-modes [6].

It has been shown in Ref. 77] that such density fluctuations can affect neutrino propagation in an important way. The effect of random matter density fluctuations on the electron-neutrino survival probability for LMA oscillations has been shown to be sizable if the correlation length $L_{0}$ (we take $L_{0}=100 \mathrm{~km}$ ) is comparable to the neutrino oscillation length in the Sun. This is illustrated in Fig. 8. The fluctuation's amplitude $\xi$ at the position of neutrino resonance is zero in the left panel, and is $\xi=4 \%$ and $\xi=8 \%$ in the middle and right panels, respectively. The corresponding solar neutrino oscillation parameters obtained in our global fit are shown in Fig. 9] before and after the new KamLAND data presented at Neutrino 2004. One sees that these new data have a rather strong impact on the stability of the oscillation parameters. With 2002 KamLAND data an additional allowed region was present for $\Delta m_{\mathrm{SOL}}^{2} \sim 2 \times 10^{-5} \mathrm{eV}^{2}$ at the $99 \%$ C.L. [78], whereas the new KamLAND data pin down the oscillation parameters such that the allowed regions in Fig. 9 are practically stable, and the new solar noiseinduced solution appears only marginally at the $3 \sigma$ confidence level.

Conversely, as shown in Ref. 78], the quality of current solar neutrino measurements after the SNO-salt and KamLAND results is sufficiently good as to place important constraints on fluctuations in the solar medium deep within the solar radiative zone. In other words, neutrinos may be used as an astrophysical probe of the solar interior, beyond the framework of the Standard Solar Model. As illustrated in Fig. 10 density 


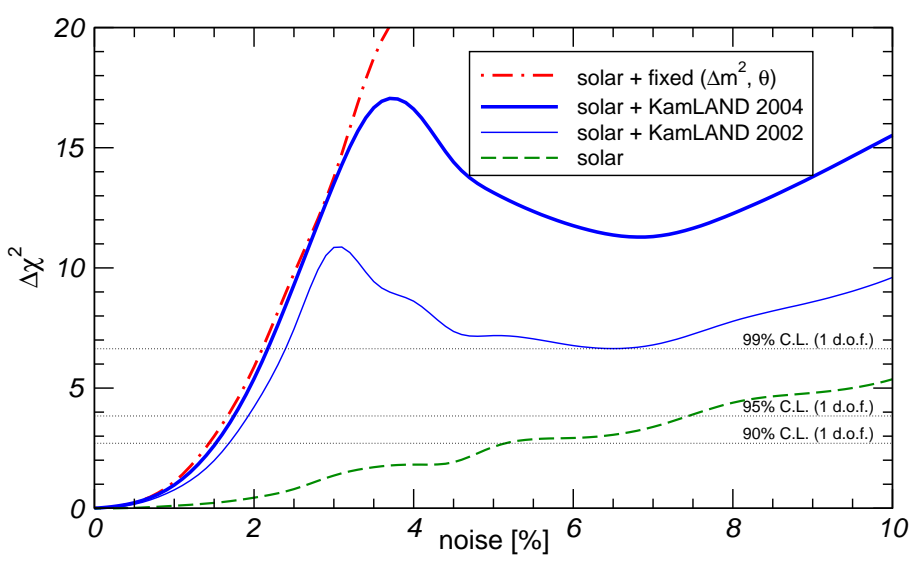

Figure 10. Bounds on random matter density fluctuations for a correlation length of $L_{0}=100 \mathrm{~km}$ from solar neutrino data, solar + KamLAND data, and solar data for oscillation parameters fixed at the best fit point.

fluctuations are strongly constrained if the correlation length lies in the range of several hundred $\mathrm{km}$. Comparing the curves for free and fixed oscillation parameters one notes that the bounds on fluctuations have already become rather stable, a situation that may still improve when the accuracy on $\Delta m_{\mathrm{SOL}}^{2}$ and $\theta_{\mathrm{SOL}}$ gets improved by future neutrino experiments. Because oscillations are sensitive to correlation lengths which are so short, such solar neutrino results will complement the constraints that come from helioseismology.

3.4.2. Beyond solar neutrino oscillations: Spin Flavour Precession In extensions of the Standard Model (SM) neutrino masses are in general accompanied also by nonstandard interactions and/or electromagnetic properties. In the minimal extension of the Standard Model with Dirac neutrino masses one expects very tiny neutrino magnetic moments (MMs) 79], well below current experimental sensitivities. However, the theoretically preferred case of Majorana neutrinos leads to potentially larger transition magnetic moments closer to the present sensitivities. These can affect neutrino propagation properties in the Sun beyond the oscillation mechanism, due to the possible presence of solar magnetic fields. Alternatively, they can affect the determination of neutrino oscillation parameters due to non-standard neutrino cross sections inside the detectors.

The most general form of the electromagnetic current of massive (Majorana) neutrinos has been given in Ref. 80]. The magnetic piece is characterized by a $3 \times 3$ complex antisymmetric matrix, the so-called Majorana transition moment (TM) matrix, that contains MMs as well as electric dipole moments of the neutrinos. Their existence would affect neutrino propagation inside the solar convective zone due to a spin-flavour precession (SFP) effect [80, 81, 82]. This in general depends on the assumed magnetic field profile. In order to quantify the extent with which the oscillation regions can be altered by the sub-leading spin-flavour precession effect, a $\chi^{2}$ analysis was performed 
in Ref. 83] taking into account the global solar + KamLAND disappearance data. We assumed that neutrino conversions are driven mainly by LMA oscillations, and used the same self-consistent [84] convective-zone solar magnetic field profile employed in Ref. [85]. The results we obtain indicate that, even though small, current bounds on neutrino magnetic moments and solar magnetic fields still leave room for slight modifications in the determinations of solar neutrino oscillation parameters, in the presence of large magnetic moments $\boldsymbol{\top}$.

However, in the general Majorana case, where theory may give rise to higher moments, there is a characteristic feature of the spin flavour precession which will lead to more stringent constraints, and hence increase the robustness of the oscillation parameter determination. The argument is based on the presence of anti-neutrinos in the solar flux [80, 81, 82]. Recently the KamLAND collaboration 86] has reported a result which greatly improves the bound on an anti-neutrino component in the solar flux from $0.1 \%$ of the solar boron $\nu_{e}$ flux to $2.8 \times 10^{-2} \%$ at the $90 \%$ C.L., about 30 times better than the recent Super-K limit [87]. This implies that, in practical terms, extremely good stability of the solar neutrino oscillation parameters against the possible existence of sub-leading SFP conversions is obtained. As a result, we conclude that solar neutrinos oscillation parameters can be inferred without any reference to neutrino magnetic properties nor solar magnetic fields. We refer the reader to Ref. [83] for quantitative details.

All in all, our analysis of solar neutrino data implies indicates pretty good stability of the oscillation parameter determination for the case of Majorana neutrinos, due to the solar anti-neutrino limit from KamLAND. In contrast for the special case of Dirac neutrinos this limit does not apply and the determination of oscillation parameters is potentially more fragile. However, we note that the gauge theoretic expectations for Dirac magnetic moments are typically lower than those for Majorana neutrino transition moments.

\subsubsection{Beyond SM neutrino cross sections: Constraining neutrino magnetic moments} Neutrino transition magnetic moments are basic properties of neutrinos 80]. Although they do not substantially affect neutrino propagation, even in the presence of solar magnetic fields, non-trivial electromagnetic neutrino properties could still show up in the detection process and to this extent affect the determination of oscillation parameters. Experiments based on the neutrino detection via neutrino-electron elastic scattering are a sensitive probe of the electromagnetic properties. In Ref. 88] it was shown that current data from solar neutrinos (in particular from Super-K) in combination with reactor neutrino-electron scattering data provides strong bounds on all the elements of the TM matrix (for similar analyses see Ref. [89, 90, 91, 92]).

In several experiments such as Super-K, Borexino and some reactor experiments [93,
94, 95], neutrinos are detected via the elastic neutrino-electron scattering, whose

9 Note that our analysis of solar neutrino data applies also to the special case of Dirac neutrinos. 
electromagnetic cross section is 96,97

$$
\frac{d \sigma_{\mathrm{em}}}{d T}=\frac{\alpha^{2} \pi}{m_{e}^{2} \mu_{B}^{2}}\left(\frac{1}{T}-\frac{1}{E_{\nu}}\right) \mu_{\mathrm{eff}}^{2},
$$

where $\mu_{\text {eff }}$ is an effective MM 98], $T$ denotes the kinetic energy of the recoil electron and $E_{\nu}$ is the incoming neutrino energy. The electromagnetic cross section adds to the weak cross section and allows to extract information on the TM matrix, which we denote by $\lambda$ in the following. Taking into account the antisymmetry of $\lambda$ for Majorana neutrinos, it is useful to define vectors $\Lambda$ by $\lambda_{j k}=\varepsilon_{j k l} \Lambda_{l}$, where $\lambda_{j k}$ are the elements of the TM matrix in the mass basis. The effective MM square $\mu_{\text {eff }}^{2}$ takes on different forms in the cases of solar and reactor neutrino experiments. The detailed derivation of the following expressions can be found in Ref. [88]. For the case of solar neutrino experiments one obtains the effective MM square

$$
\mu_{\mathrm{LMA}}^{2}=|\boldsymbol{\Lambda}|^{2}-\left|\Lambda_{2}\right|^{2}+P_{e 1}^{2 \nu}\left(\left|\Lambda_{2}\right|^{2}-\left|\Lambda_{1}\right|^{2}\right),
$$

where $P_{e 1}^{2 \nu}$ corresponds to the probability that an electron neutrino produced in the core of the sun arrives at the detector as the mass eigenstate $\nu_{1}$ in a two-neutrino scheme. In contrast, the $\mu_{\text {eff }}^{2}$ relevant in reactor experiments is given as

$$
\mu_{\mathrm{R}}^{2}=|\boldsymbol{\Lambda}|^{2}-\cos ^{2} \theta_{\mathrm{SOL}}\left|\Lambda_{1}\right|^{2}-\sin ^{2} \theta_{\mathrm{SOL}}\left|\Lambda_{2}\right|^{2}-\sin 2 \theta_{\mathrm{SOL}}\left|\Lambda_{1}\right|\left|\Lambda_{2}\right| \cos \delta,
$$

where $\delta=\arg \left(\Lambda_{1}^{*} \Lambda_{2}\right)$ is the relative phase between $\Lambda_{1}$ and $\Lambda_{2}$.

In the following we discuss the constraints on neutrino TMs from solar and reactor neutrino experiments [88]. The $\chi^{2}$ obtained from the data is minimized with respect to all TM parameters except the modulus $|\boldsymbol{\Lambda}|$. To take into account the physical boundary $|\boldsymbol{\Lambda}| \geq 0$ we use Bayesian methods to calculate an upper bound on $|\boldsymbol{\Lambda}|$. Let us stress that these bounds apply to all elements of the TM matrix, including MMs and electric dipole moments of all neutrino flavours, since $|\boldsymbol{\Lambda}|^{2}=\left|\Lambda_{1}\right|^{2}+\left|\Lambda_{2}\right|^{2}+\left|\Lambda_{3}\right|^{2}$. Furthermore, since $|\boldsymbol{\Lambda}|$ is independent of the basis these bounds apply also for the TMs in the flavour basis.

In the left panel of Fig. 11] we show contours of the $90 \%$ C.L. bound on $|\boldsymbol{\Lambda}|$ in the $\left(\tan ^{2} \theta_{\mathrm{SOL}}, \Delta m_{\mathrm{SOL}}^{2}\right)$ plane for the combination of solar and reactor data. We note that in the upper parts of the LMA region, the solar data alone give already a strong bound on $|\boldsymbol{\Lambda}|$, see Ref. [88] for details. In contrast, for low $\Delta m_{\mathrm{SOL}}^{2}$ values the inclusion of reactor data plays an important role in improving the bound. From our analysis we find at $90 \%$ C.L.:

$$
|\boldsymbol{\Lambda}|< \begin{cases}3.4 \times 10^{-10} \mu_{B} & (\text { solar }+ \text { KamLAND data }) \\ 1.7 \times 10^{-10} \mu_{B} & (\text { solar }+ \text { KamLAND + reactor data })\end{cases}
$$

where for each value of $|\boldsymbol{\Lambda}|$ we have minimized the $\chi^{2}$ with respect to $\tan ^{2} \theta_{\text {SOL }}$ and $\Delta m_{\mathrm{SOL}}^{2}$.

Finally we note that the Borexino experiment [99] will improve these bounds roughly by one order of magnitude. This experiment is mainly sensitive to the solar ${ }^{7} \mathrm{Be}$ neutrino flux, which will be measured by elastic neutrino-electron scattering. Therefore, 

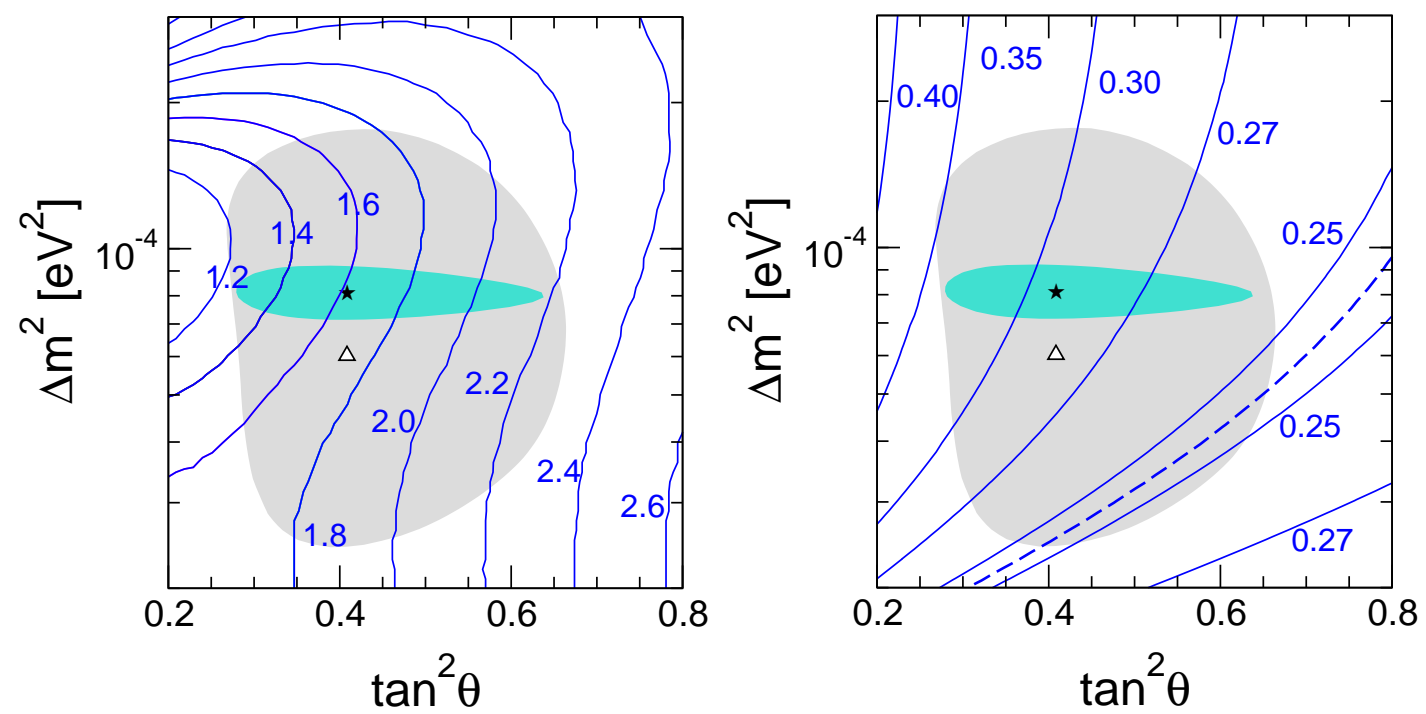

Figure 11. Contours of the $90 \%$ C.L. bound on $|\boldsymbol{\Lambda}|$ in units of $10^{-10} \mu_{B}$ from combined solar and reactor data (left panel) and after 3 years of Borexino data-taking (right panel). The gray (light) shaded region is the $3 \sigma$ LMA region obtained in the global analysis of solar neutrino data (best fit point marked with a triangle), whereas the green (dark) one corresponds to the $3 \sigma$ region obtained after including the KamLAND results (best fit point marked with a star). The dashed line in the right panel corresponds to $P_{e 1}=0.5$ for ${ }^{7}$ Be neutrinos, and shows the strongest attainable limit.

Borexino is similar to Super-K, the main difference is the mono-energetic line of the ${ }^{7} \mathrm{Be}$ neutrinos, with an energy of $0.862 \mathrm{MeV}$, which is roughly one order of magnitude smaller than the energies of the ${ }^{8} \mathrm{~B}$ neutrino flux relevant in Super-K. Thanks to the lower neutrino energy the sensitivity to electromagnetic properties is increased, as can be seen from Eq. (10). Details about our Borexino simulation can be found in Ref. 88]. At the best fit point one finds the sensitivity

$$
|\boldsymbol{\Lambda}| \leq 0.29 \times 10^{-10} \mu_{B} \quad \text { at } 90 \% \text { C.L. }
$$

after three years of Borexino data taking. In the right panel of Fig. [1] we show contours of the $90 \%$ C.L. bound in the $\left(\tan ^{2} \theta_{\mathrm{SOL}}, \Delta m_{\mathrm{SOL}}^{2}\right)$ plane.

\section{Three-flavour neutrino oscillations}

\subsection{Global three-neutrino analysis}

In this section the three-neutrino oscillation parameters are determined from a global analysis of the most recent neutrino oscillation data. For earlier three-neutrino analyses see Refs. [35, 100, 101]. To fix the notation, we define the neutrino mass-squared differences $\Delta m_{\mathrm{SOL}}^{2} \equiv \Delta m_{21}^{2} \equiv m_{2}^{2}-m_{1}^{2}$ and $\Delta m_{\mathrm{ATM}}^{2} \equiv \Delta m_{31}^{2} \equiv m_{3}^{2}-m_{1}^{2}$, and use the convenient form of the parameterization for the leptonic mixing matrix given in Ref. 20] 


\begin{tabular}{|l|c|c|c|c|}
\hline parameter & best fit & $2 \sigma$ & $3 \sigma$ & $4 \sigma$ \\
\hline$\Delta m_{21}^{2}\left[10^{-5} \mathrm{eV}^{2}\right]$ & 8.1 & $7.5-8.7$ & $7.2-9.1$ & $7.0-9.4$ \\
$\Delta m_{31}^{2}\left[10^{-3} \mathrm{eV}^{2}\right]$ & 2.2 & $1.7-2.9$ & $1.4-3.3$ & $1.1-3.7$ \\
$\sin ^{2} \theta_{12}$ & 0.30 & $0.25-0.34$ & $0.23-0.38$ & $0.21-0.41$ \\
$\sin ^{2} \theta_{23}$ & 0.50 & $0.38-0.64$ & $0.34-0.68$ & $0.30-0.72$ \\
$\sin ^{2} \theta_{13}$ & 0.000 & $\leq 0.028$ & $\leq 0.047$ & $\leq 0.068$ \\
\hline
\end{tabular}

Table 1. Best-fit values, $2 \sigma, 3 \sigma$, and $4 \sigma$ intervals (1 d.o.f.) for the three-flavour neutrino oscillation parameters from global data including solar, atmospheric, reactor (KamLAND and CHOOZ) and accelerator (K2K) experiments.

and now adopted as standard by the PDG [102]:

$$
U=\left(\begin{array}{ccc}
c_{13} c_{12} & s_{12} c_{13} & s_{13} \\
-s_{12} c_{23}-s_{23} s_{13} c_{12} & c_{23} c_{12}-s_{23} s_{13} s_{12} & s_{23} c_{13} \\
s_{23} s_{12}-s_{13} c_{23} c_{12} & -s_{23} c_{12}-s_{13} s_{12} c_{23} & c_{23} c_{13}
\end{array}\right),
$$

where $c_{i j} \equiv \cos \theta_{i j}$ and $s_{i j} \equiv \sin \theta_{i j}$. Furthermore, we use the notations $\theta_{12} \equiv \theta_{\text {SoL }}$ and $\theta_{23} \equiv \theta_{\text {ATM }}$. Because of the hierarchy $\Delta m_{\mathrm{SOL}}^{2} \ll \Delta m_{\mathrm{ATM}}^{2}$ it is a good approximation to set $\Delta m_{\mathrm{SOL}}^{2}=0$ in the analysis of atmospheric and $\mathrm{K} 2 \mathrm{~K}$ data ${ }^{+}$, and to set $\Delta m_{\mathrm{ATM}}^{2}$ to infinity for the analysis of solar and KamLAND data. This implies furthermore that the effect of a possible Dirac CP-violating phase [20] in the lepton mixing matrix can be neglected $*$. We perform a general fit to the global data in the five-dimensional parameter space $s_{12}^{2}, s_{23}^{2}, s_{13}^{2}, \Delta m_{21}^{2}, \Delta m_{31}^{2}$, and show projections onto various one- or two-dimensional sub-spaces.

We include in our analysis the global solar neutrino oscillation data from all solar neutrino experiments and the KamLAND reactor experiment as described in Sec. 3, the atmospheric neutrino data from Super-K, as well as spectral data from the K2K long-baseline experiment (see Sec. 21). In addition we take into account in our fit the constraints from the CHOOZ reactor experiment [105].

The results of the global three-neutrino analysis are summarized in Fig. 12 and in Tab. 1. In the upper panels of the figure the $\Delta \chi^{2}$ is shown as a function of the parameters $\sin ^{2} \theta_{12}, \sin ^{2} \theta_{23}, \sin ^{2} \theta_{13}, \Delta m_{21}^{2}, \Delta m_{31}^{2}$, minimized with respect to the undisplayed parameters. The lower panels show two-dimensional projections of the allowed regions in the five-dimensional parameter space. The best fit values and the allowed ranges of the oscillation parameters from the global data are given in Tab. 1. This table summarizes the current status of the three-flavour neutrino oscillation parameters.

+ See Ref. 103 for a two-mass scale analysis of atmospheric data.

* The two Majorana phases [20] do not show up in oscillations but do appear in lepton number violating processes 104]. 

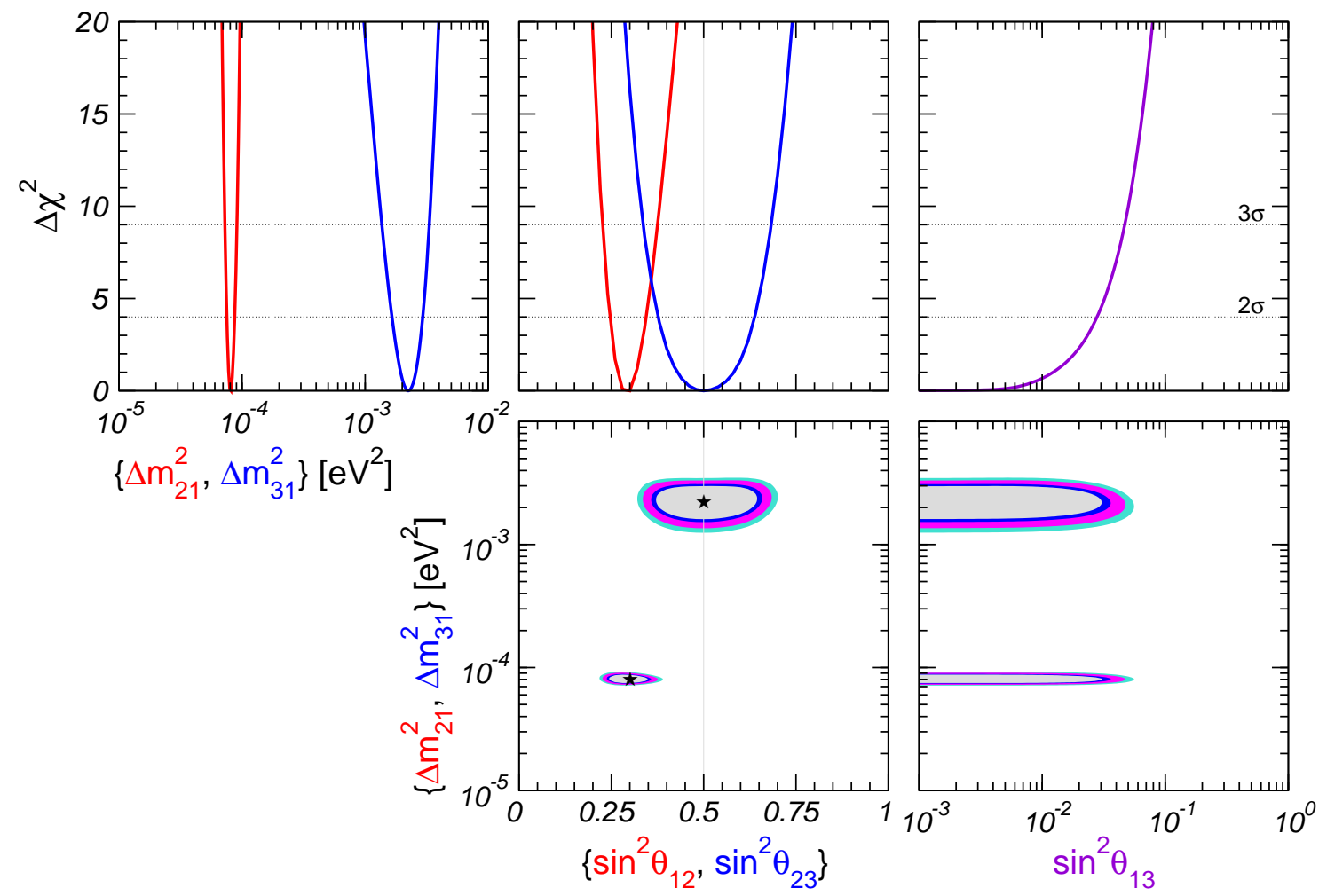

Figure 12. Projections of the allowed regions from the global oscillation data at $90 \%$, $95 \%, 99 \%$, and $3 \sigma$ C.L. for 2 d.o.f. for various parameter combinations. Also shown is $\Delta \chi^{2}$ as a function of the oscillation parameters $\sin ^{2} \theta_{12}, \sin ^{2} \theta_{23}, \sin ^{2} \theta_{13}, \Delta m_{21}^{2}, \Delta m_{31}^{2}$, minimized with respect to all undisplayed parameters.

\subsection{The small parameters $\alpha \equiv \Delta m_{\mathrm{SOL}}^{2} / \Delta m_{\mathrm{ATM}}^{2}$ and $\theta_{13}$}

Genuine three-flavour effects are associated to the mass hierarchy parameter $\alpha \equiv$ $\Delta m_{\mathrm{SOL}}^{2} / \Delta m_{\mathrm{ATM}}^{2}$ and the mixing angle $\theta_{13}$. In particular, in a three-neutrino scheme $\mathrm{CP}$ violation disappears in the limit where two neutrinos become degenerate [20, 106] and in the limit where $\theta_{13} \rightarrow 0$. We discuss in this subsection the present status of these small parameters.

In Fig. 13 the $\Delta \chi^{2}$ from the global data is shown as a function of the mass hierarchy parameter $\alpha$. Also shown in this figure is the $\Delta \chi^{2}$ as a function of the parameter combination $\alpha \sin 2 \theta_{12}$, since to leading order in the long baseline $\nu_{e} \rightarrow \nu_{\mu}$ oscillation probability solar parameters appear in this particular combination [107, 108]. We obtain the following best fit values and $3 \sigma$ intervals:

$$
\begin{array}{ll}
\alpha=0.035, & 0.024 \leq \alpha \leq 0.060, \\
\alpha \sin 2 \theta_{12}=0.032, & 0.022 \leq \alpha \sin 2 \theta_{12} \leq 0.054 .
\end{array}
$$

Let us now discuss the status of the mixing angle $\theta_{13}$, which at the moment is the last unknown angle in the three-neutrino leptonic mixing matrix. Only an upper bound exists, which used to be dominated by the CHOOZ [105] and Palo Verde [109] reactor experiments. Currently a large effort is put to determine this angle in future 


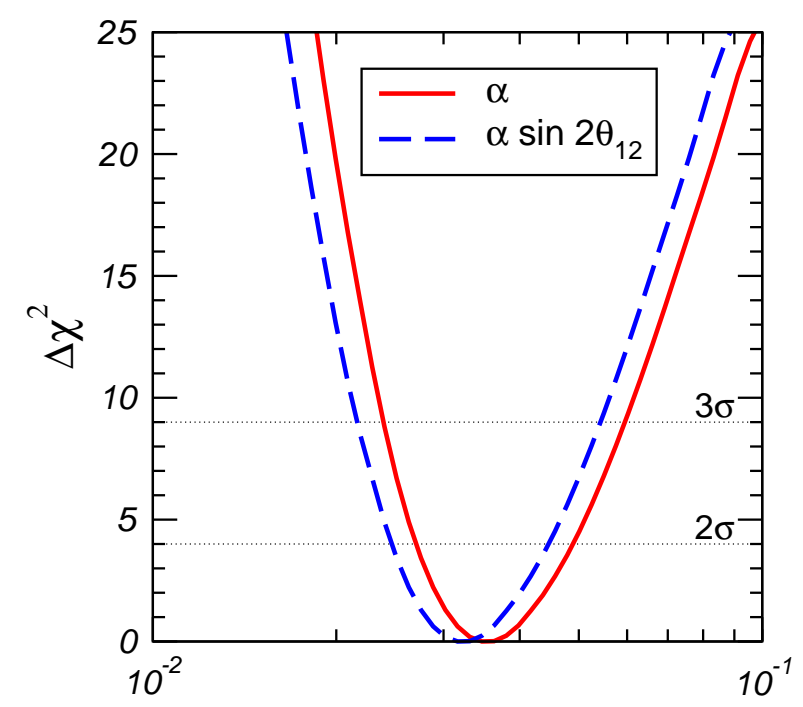

Figure 13. $\Delta \chi^{2}$ from global oscillation data as a function of $\alpha \equiv \Delta m_{\mathrm{SOL}}^{2} / \Delta m_{\mathrm{ATM}}^{2}$ and $\alpha \sin 2 \theta_{12}$.

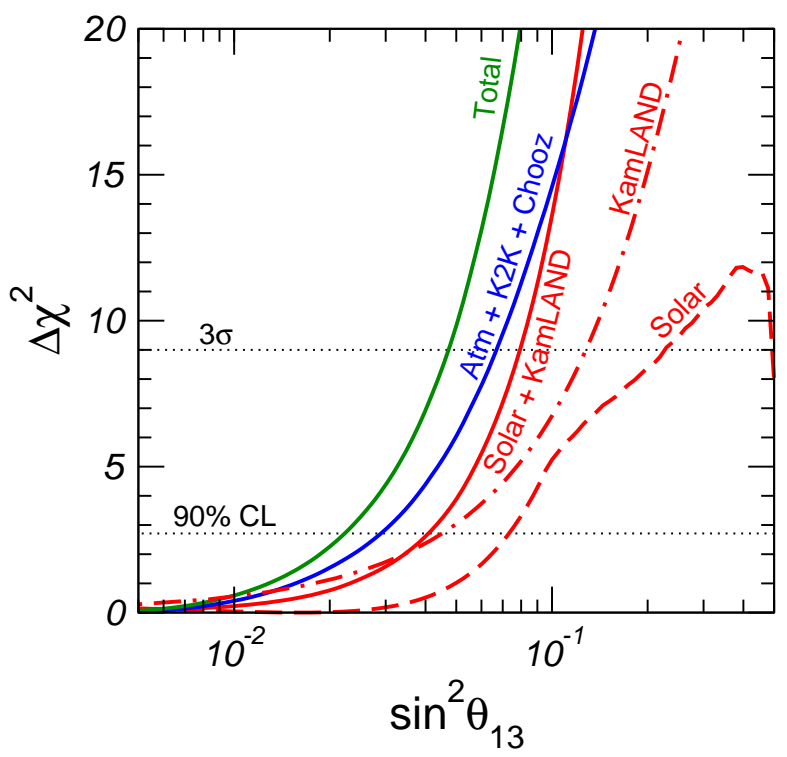

Figure 14. $\Delta \chi^{2}$ profiles projected onto the $\sin ^{2} \theta_{13}$ axis, for solar, KamLAND, solar+KamLAND, atmospheric $+\mathrm{K} 2 \mathrm{~K}+\mathrm{CHOOZ}$, and for the global data.

experiments (see, e.g., Refs. [26, 27, 40]).

In Fig. 14 we show the $\Delta \chi^{2}$ as a function of $\sin ^{2} \theta_{13}$ for different data sample choices. From this figure we find the following bounds at $90 \%$ C.L. $(3 \sigma)$ for 1 d.o.f.:

$$
\sin ^{2} \theta_{13} \leq \begin{cases}0.041(0.079) & (\text { solar }+ \text { KamLAND }) \\ 0.029(0.067) & \text { (CHOOZ+atmospheric }+\mathrm{K} 2 \mathrm{~K}) \\ 0.022(0.047) & \text { (global data) }\end{cases}
$$

We find that the new data from KamLAND have a surprisingly strong impact on this bound. Before the 2004 KamLAND data the bound on $\sin ^{2} \theta_{13}$ from global data was dominated by the $\mathrm{CHOOZ}$ reactor experiment, together with the determination of 


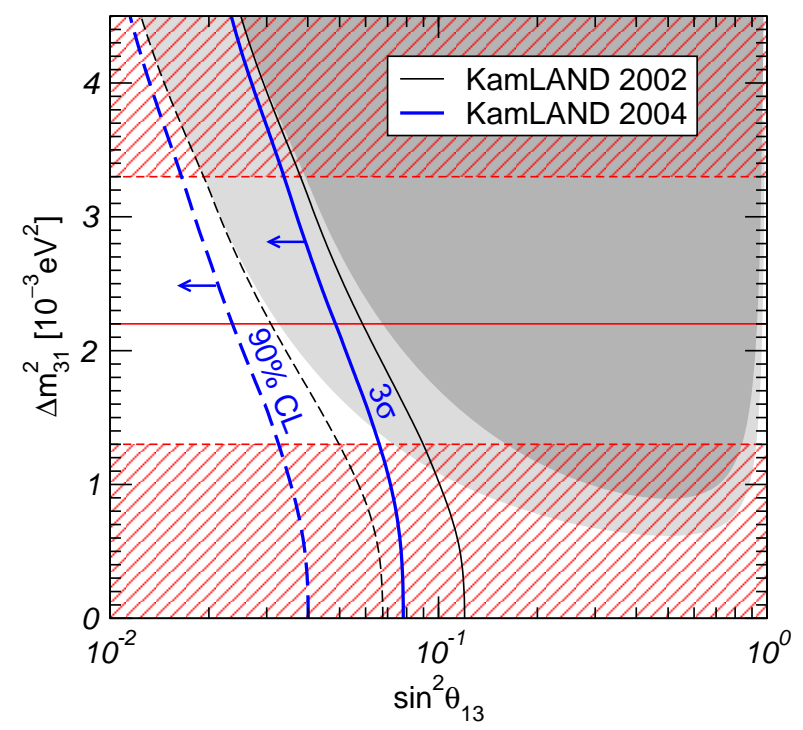

Figure 15. Upper bound on $\sin ^{2} \theta_{13}$ (1 d.o.f.) from solar+KamLAND + CHOOZ data as a function of $\Delta m_{\mathrm{ATM}}^{2}$. The dashed (solid) curve corresponds to the $90 \%(3 \sigma)$ C.L. bound, the thin curves have been obtained with 2002 KamLAND data, whereas the thick curves follow from the recent 2004 KamLAND update. The light (dark) shaded region is excluded by $\mathrm{CHOOZ}$ data alone at $90 \%(3 \sigma)$ C.L. The horizontal line corresponds to the best fit value of $\Delta m_{\mathrm{ATM}}^{2}$ from atmospheric $+\mathrm{K} 2 \mathrm{~K}$ data given in Eq. (2), and the hatched regions are excluded by atmospheric $+\mathrm{K} 2 \mathrm{~K}$ data at $3 \sigma$ according to Eq. (3).

$\Delta m_{31}^{2}$ from atmospheric data (see e.g., Ref. [30]). However, using most recent data the combined bound from solar+KamLAND becomes comparable to the CHOOZ bound, and these data contribute notably to the final bound. A detailed discussion of the reason for such improvement on $\sin ^{2} \theta_{13}$ from the 2004 KamLAND data is given in Appendix A.2. One reason is the rather strong signal for spectral distortion in the current sample.

As noted in Ref. 30] the bound from solar+KamLAND is especially important for the relatively lower values of $\Delta m_{\mathrm{ATM}}^{2}$ implied by the use of the new three-dimensional atmospheric fluxes [32], since the CHOOZ bound on $\sin ^{2} \theta_{13}$ deteriorates quickly when $\Delta m_{\text {ATM }}^{2}$ decreases (see Fig. 15). Such loosening in sensitivity for low $\Delta m_{\text {Aтм }}^{2}$ values is prevented first, by the lower bound on $\Delta m_{\mathrm{ATM}}^{2}$ from K2K (see Fig. 21) and second, by the bound from solar+KamLAND, which is independent of $\Delta m_{\mathrm{ATM}}^{2}$. In Fig. 15 we show the upper bound on $\sin ^{2} \theta_{13}$ as a function of $\Delta m_{\mathrm{ATM}}^{2}$ from CHOOZ data alone compared to the bound from an analysis including solar and reactor neutrino data (CHOOZ and

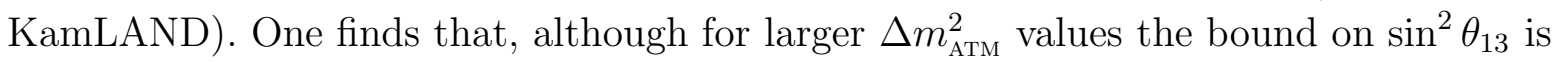
dominated by the CHOOZ data, for $\Delta m_{\mathrm{ATM}}^{2} \lesssim 2 \times 10^{-3} \mathrm{eV}^{2}$ the solar + KamLAND data start being important. For illustration we show in Fig. 15 also the bound as implied by the old 2002 KamLAND data to highlight the improvement of the new data. We note that, as before, the bound from solar data is rather stable under the recent SSM update. 


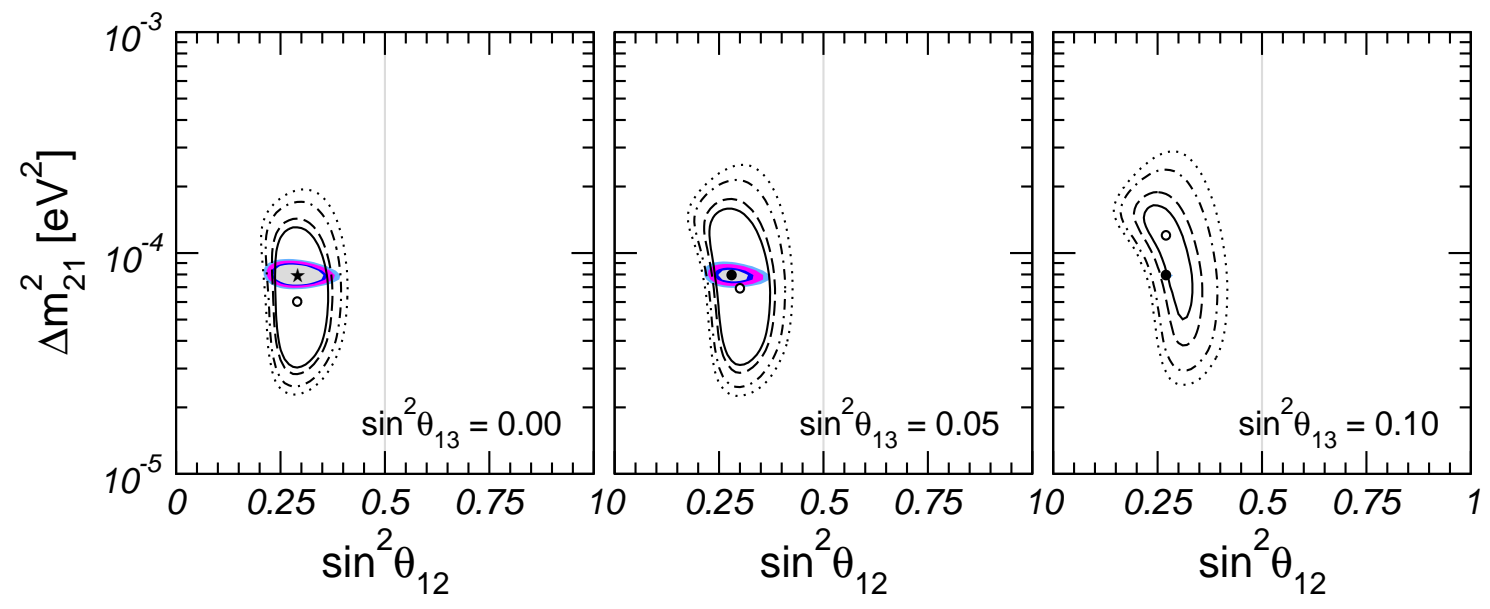

Figure 16. Sections of the three-dimensional allowed regions in the $\left(\sin ^{2} \theta_{\mathrm{SOL}}, \Delta m_{\mathrm{SOL}}^{2}\right)$ plane at $90 \%, 95 \%, 99 \%$ and $3 \sigma$ C.L. for 3 d.o.f. for various $\sin ^{2} \theta_{13}$ values from solar data (lines) and solar+KamLAND data (colored regions). The local minima in each plane from solar+KamLAND (solar only) data are marked by filled (open) dots.

Let us discuss in some more detail the constraint on $\sin ^{2} \theta_{13}$ from solar data, which emerges from a subtle interplay of various solar neutrino observables. In Fig. 16] we show the results of a three parameter fit $\left(\sin ^{2} \theta_{\mathrm{SOL}}, \Delta m_{\mathrm{SOL}}^{2}, \sin ^{2} \theta_{13}\right)$ to solar and KamLAND data. Allowed regions are shown for various values of $\sin ^{2} \theta_{13}$ in the $\left(\sin ^{2} \theta_{\mathrm{SOL}}, \Delta m_{\mathrm{SOL}}^{2}\right)$ plane with respect to the global minimum. Note that here we calculate the allowed regions at a given confidence level for 3 d.o.f. The shape of $\Delta \chi^{2}$ from solar data shown in Fig. 14 can be understood from Fig. 16. Indeed one observes that for solar data increasing $\theta_{13}$ can be compensated to some extent by increasing $\Delta m_{\mathrm{SOL}}^{2}$. Since solar data disfavours large values of $\Delta m_{\mathrm{SOL}}^{2}$ the bound improves. Also the combination with KamLAND has a similar effect, since recent KamLAND data essentially fix $\Delta m_{\mathrm{SOL}}^{2}$ at roughly $8 \times 10^{-5} \mathrm{eV}^{2}$ such that the continuous rise of $\Delta m_{\mathrm{SOL}}^{2}$ with $\sin ^{2} \theta_{13}$ preferred by solar data is prevented. On the other hand solar data breaks a correlation of $\sin ^{2} \theta_{13}$ and $\sin ^{2} \theta_{12}$ in the KamLAND data (see Appendix A.2), which again leads to an improvement of the combined bound on $\sin ^{2} \theta_{13}$.

The difference in the day/night solar neutrino fluxes due to the regeneration effect in the earth in the three-flavour framework has been considered recently in Refs. [110, 111]. This observable may provide valuable information on $\theta_{13}$ in the context of future solar neutrino experiments like UNO or Hyper-K [112].

\section{Four-neutrino oscillations and LSND}

In addition to the strong evidence for oscillations due to the mass-squared differences $\Delta m_{\mathrm{SOL}}^{2}$ and $\Delta m_{\mathrm{ATM}}^{2}$ there is also a hint for oscillations with a much larger mass-squared difference from the LSND experiment [113]. This accelerator experiment performed at Los Alamos observed 87.9 $\pm 22.4 \pm 6.0$ excess events in the $\bar{\nu}_{\mu} \rightarrow \bar{\nu}_{e}$ appearance channel, corresponding to a transition probability of $P=(0.264 \pm 0.067 \pm 0.045) \%$, which is 

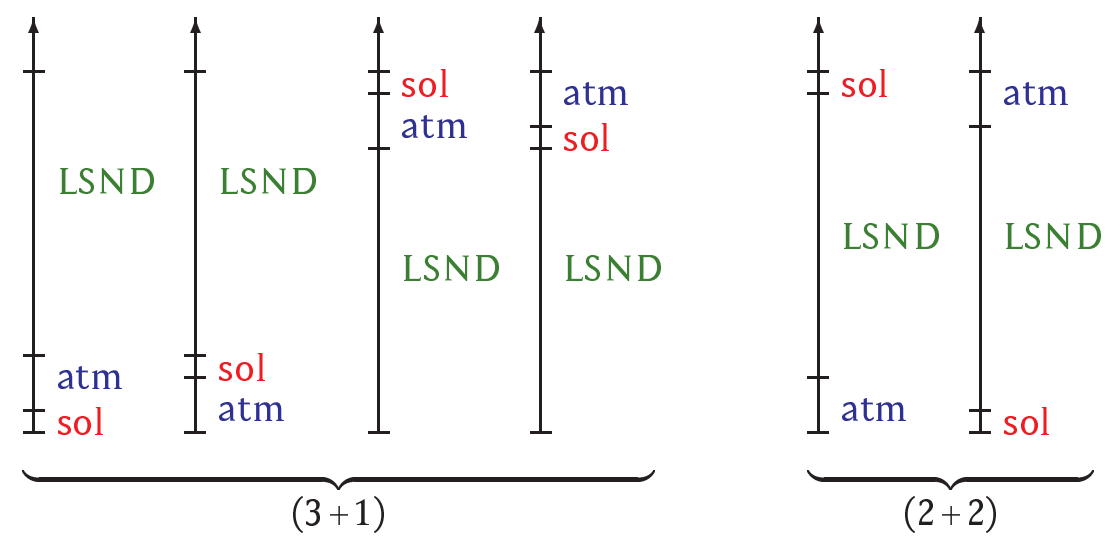

Figure 17. The two classes of six four-neutrino mass spectra, $(3+1)$ and $(2+2)$.

$\sim 3.3 \sigma$ away from zero. To explain this signal with neutrino oscillations requires a mass-squared difference $\Delta m_{\mathrm{LSND}}^{2} \sim 1 \mathrm{eV}^{2}$. Such a value is inconsistent with the masssquared differences required by solar/KamLAND and atmospheric/K2K experiments within the standard three-flavour framework. In this section we consider four-neutrino schemes, where a sterile neutrino [114, 115, 116] is added to the three active ones to provide the additional mass scale needed to reconcile the LSND evidence. We include in our analysis data from the LSND experiment, as well as from short-baseline (SBL) accelerator [117, 118] and reactor [105, 109, 119] experiments reporting no evidence for oscillations (see Ref. [120] for details of our SBL data analysis). We update our previous four-neutrino analyses (see, e.g., Refs. 121, 122]) by including the most recent solar and KamLAND [15] data, the improved atmospheric neutrino fluxes [32] and latest data from the K2K long-baseline experiment [17].

\subsection{A common parameterization for four-neutrino schemes}

Four-neutrino mass schemes are usually divided into the two classes $(3+1)$ and $(2+2)$, as illustrated in Fig. 17. We note that $(3+1)$ mass spectra include the three-active neutrino scenario as limiting case. In this case solar and atmospheric neutrino oscillations are explained by active neutrino oscillations, with mass-squared differences $\Delta m_{\mathrm{SOL}}^{2}$ and $\Delta m_{\mathrm{ATM}}^{2}$, and the fourth neutrino state gets completely decoupled. We will refer to such limiting scenario as $(3+0)$. In contrast, the $(2+2)$ spectrum is intrinsically different, as the sterile neutrino must take part in either solar or in atmospheric neutrino oscillations, or in both.

Neglecting CP violation, neutrino oscillations in four-neutrino schemes are generally described by 9 parameters: 3 mass-squared differences and 6 mixing angles in the lepton mixing matrix [20]. We use the parameterization introduced in Ref. [123], in terms of $\Delta m_{\mathrm{SOL}}^{2}, \theta_{\mathrm{SOL}}, \Delta m_{\mathrm{ATM}}^{2}, \theta_{\mathrm{ATM}}, \Delta m_{\mathrm{LSND}}^{2}, \theta_{\mathrm{LSND}}$. These 6 parameters are similar to the two-neutrino mass-squared differences and mixing angles and are directly related to the oscillations in solar, atmospheric and the LSND experiments. For the remaining 3 


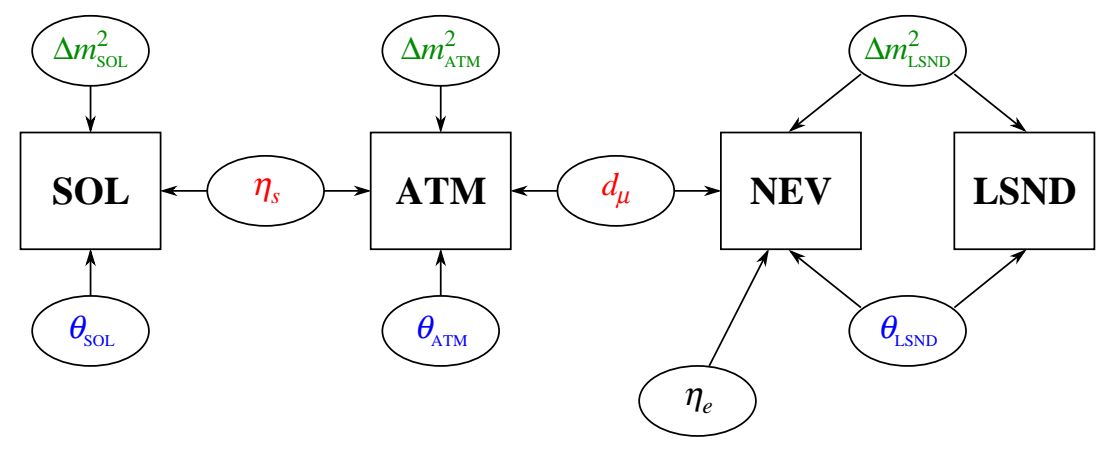

Figure 18. Parameter dependence of the different data sets in our parameterization.

parameters we use $\eta_{s}, \eta_{e}$ and $d_{\mu}$. These quantities are defined by

$$
\begin{aligned}
& \eta_{\alpha}=\sum_{i}\left|U_{\alpha i}\right|^{2} \quad \text { with } i \in \text { solar mass states, } \\
& d_{\alpha}=1-\sum_{i}\left|U_{\alpha i}\right|^{2} \quad \text { with } i \in \text { atmospheric mass states, }
\end{aligned}
$$

where $\alpha=e, \mu, \tau, s$. Note that in $(2+2)$ schemes the relation $\eta_{\alpha}=d_{\alpha}$ holds, whereas in $(3+1) \eta_{\alpha}$ and $d_{\alpha}$ are independent. The physical meaning of these parameters is the following: $\eta_{\alpha}$ is the fraction of $\nu_{\alpha}$ participating in solar oscillations, and $\left(1-d_{\alpha}\right)$ is the fraction of $\nu_{\alpha}$ participating in oscillations with $\Delta m_{\mathrm{ATM}}^{2}$ (for further discussions see Ref. [123]). For the analysis we adopt the following approximations:

(i) We make use of the hierarchy $\Delta m_{\mathrm{SOL}}^{2} \ll \Delta m_{\mathrm{ATM}}^{2} \ll \Delta m_{\mathrm{LSND}}^{2}$. This means that for each data set we consider only one mass-squared difference, the other two are set either to zero or to infinity.

(ii) In the analyses of solar and atmospheric data (but not for SBL data) we set $\eta_{e}=1$, which is justified because of strong constraints from reactor experiments 105, 109, 119].

Within this approximation the parameter structure of the four-neutrino analysis gets rather simple. The parameter dependence of the four data sets solar, atmospheric, LSND and NEV is illustrated in Fig. [18] In this section, except where explicitly noted otherwise, we tacitly consider KamLAND as part of the solar data sample and K2K as part of the atmospheric data sample. The NEV data set contains the experiments KARMEN [17], CDHS 118], Bugey [119], CHOOZ 105], and Palo Verde [109], reporting no evidence for oscillations. We see that only $\eta_{s}$ links solar and atmospheric data and $d_{\mu}$ links atmospheric and NEV data, while LSND and NEV data are coupled by $\Delta m_{\mathrm{LSND}}^{2}$ and $\theta_{\mathrm{LSND}}$. With the definitions (18) and (19) and in our approximation the parameter structure shown in Fig. 18 holds for both types of mass spectra, $(3+1)$ as well as $(2+2)$ 123]. 

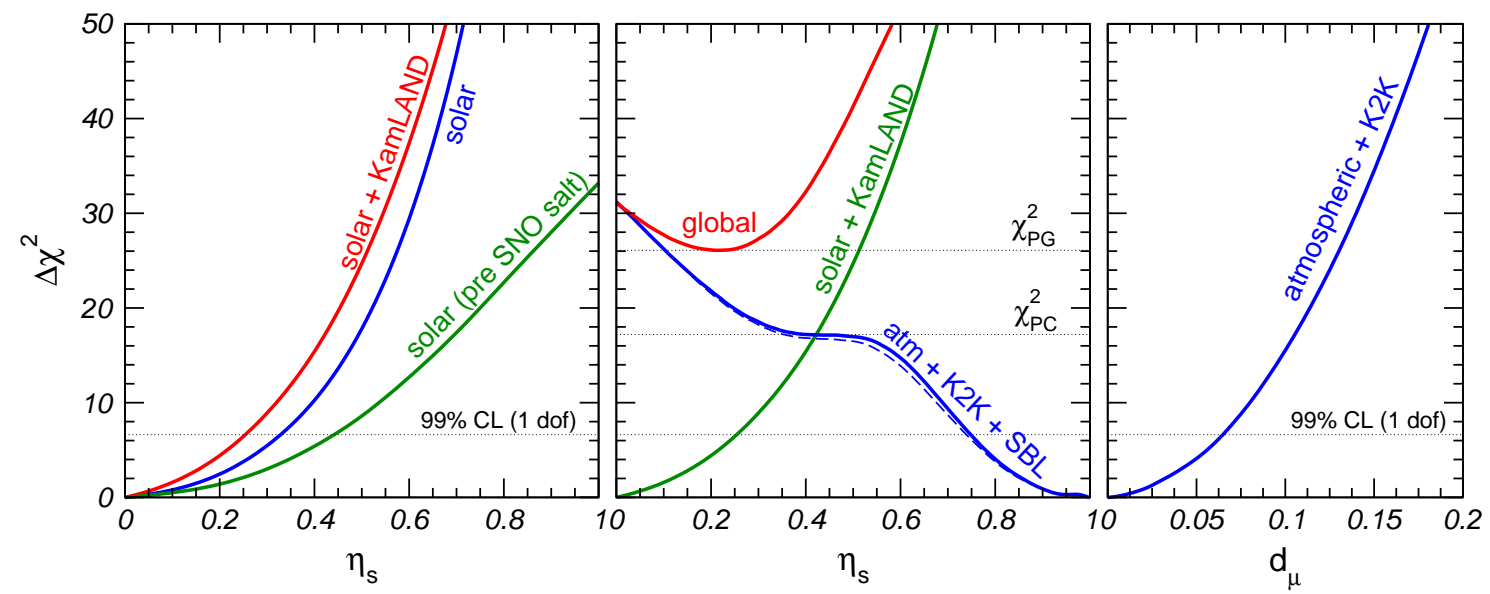

Figure 19. Left: $\Delta \chi^{2}$ as a function of $\eta_{s}$ from solar data before the SNO saltphase results, from current solar data, and from solar+KamLAND data. Middle: $\Delta \chi_{\mathrm{SOL}}^{2}, \Delta \chi_{\mathrm{ATM}+\mathrm{K} 2 \mathrm{~K}+\mathrm{SBL}}^{2}$ and $\bar{\chi}_{\text {global }}^{2}$ as a function of $\eta_{s}$ in $(2+2)$ oscillation schemes. The dashed line corresponds to atmospheric and K2K data only (without SBL data). Right: $\Delta \chi_{\mathrm{ATM}+\mathrm{K} 2 \mathrm{~K}}^{2}$ as a function of $d_{\mu}$.

\section{2. (2+2): ruled out by solar and atmospheric data}

The strong preference for oscillations into active neutrinos in solar and atmospheric oscillations [36] leads to a direct conflict in $(2+2)$ oscillation schemes. We will now show that thanks to recent solar neutrino data (in particular from the SNO-salt phase [10]) in combination with the KamLAND experiment [14], and the latest Super-K data on atmospheric neutrinos [11] the tension in the data has become so strong that $(2+2)$ oscillation schemes are essentially ruled out $\dagger$.

In the left panel of Fig. 19 we show the $\Delta \chi^{2}$ from solar neutrino data as a function of $\eta_{s}$, the parameter describing the fraction of the sterile neutrino participating in solar neutrino oscillations. It is clear from the figure that the improved determination of the neutral current event rate from the solar ${ }^{8} \mathrm{~B}$ flux implied by the salt enhanced measurement in SNO [10] substantially tightened the constraint on a sterile contribution: the $99 \%$ C.L. bound improves from from $\eta_{s} \leq 0.44$ for pre-SNO-salt to $\eta_{s} \leq 0.31$ (BP00). The boron flux predicted in the current BP04 SSM is slightly larger than the $\mathrm{NC}$ flux measured in SNO, which leaves more room for a sterile component in the solar neutrino flux. Indeed, using the BP04 SSM the bound deteriorates slightly to $\eta_{s} \leq 0.33$ at the $99 \%$ C.L. This effect illustrates that in schemes beyond minimal three-flavour oscillations the data still shows some sensitivity to the theoretical SSM input. Although KamLAND on its own is insensitive to a sterile neutrino contamination, it contributes indirectly to the bound because of the better determination of $\Delta m_{\mathrm{SOL}}^{2}$ and $\theta_{\mathrm{SOL}}$ [36]. The combined analysis leads to the $99 \%$ C.L. bound

$$
\eta_{s} \leq 0.25 \quad(\text { solar }+ \text { KamLAND, BP04). }
$$

$\dagger$ For an earlier four-neutrino analysis of solar and atmospheric data see Ref. [124]. 
In contrast, in $(2+2)$ schemes atmospheric data prefer values of $\eta_{s}$ close to 1 . From the combined analysis of Super-K atmospheric data, K2K and SBL neutrino data we obtain the bound $\eta_{s} \geq 0.75$ at $99 \%$ C.L., in clear disagreement with the bound from solar data. In the middle panel of Fig. 19 we show the $\Delta \chi^{2}$ for solar data and for atmospheric $+\mathrm{K} 2 \mathrm{~K}$ combined with SBL data as a function of $\eta_{s}$. Note that the main effect comes from atmospheric $+\mathrm{K} 2 \mathrm{~K}$ data; SBL experiments contribute only marginally, as visible from the dashed line. From this figure we also see that the "solar+KamLAND" and the "atm $+\mathrm{K} 2 \mathrm{~K}+\mathrm{SBL}$ " allowed domains overlap only at $\chi_{\mathrm{PC}}^{2}=17.2$, i.e. at the $4.1 \sigma$ level.

In the middle panel of Fig. 19] we also show the "global" $\bar{\chi}^{2}$ function defined as follows:

$$
\bar{\chi}^{2}\left(\eta_{s}\right) \equiv \Delta \chi_{\mathrm{SOL}+\mathrm{KAM}}^{2}\left(\eta_{s}\right)+\Delta \chi_{\mathrm{ATM}+\mathrm{K} 2 \mathrm{~K}+\mathrm{SBL}}^{2}\left(\eta_{s}\right) .
$$

In Refs. 121, 125] we have proposed a statistical method to evaluate the disagreement of different data sets in global analyses. The parameter goodness of fit (PG) makes use of the $\bar{\chi}^{2}$ defined in Eq. (21). This criterion is very useful to evaluate the GOF of the combination of data sets, avoiding dilution by the large number of data points, as it happens for the usual GOF criterion (for details see Ref. [125]). We find $\chi_{\mathrm{PG}}^{2} \equiv \bar{\chi}_{\min }^{2}=26.1$, which corresponds to $5.1 \sigma$. We conclude that $(2+2)$ mass schemes are ruled out by the disagreement between the latest solar and atmospheric neutrino data. This is a very robust result, independent of whether LSND is confirmed or disproved $\ddagger$.

Let us note that we now obtain a slightly smaller $\chi_{\mathrm{PG}}^{2}$ than previously [122], and the disagreement gets slightly weaker. The reason is that in the present analysis we have not included the atmospheric neutrino data from the MACRO experiment [12]. As discussed in Ref. [36], these data have some sensitivity to $\eta_{s}$ and enhance the rejection against a sterile component in atmospheric oscillations. Since relevant information to perform a consistent combined analysis of Super-K and MACRO using the new fluxes is presently not available, we prefer to use only Super-K data, which allows us to derive simpler and more robust results. $\oint$ Let us mention that we are also neglecting neutral current and tau appearance data from Super-K, which would also increase the rejection

$\ddagger$ Sub-leading effects beyond the approximations adopted here should not affect this result significantly. Allowing for additional parameters to vary at the percent level might change the ratio of some observables [126], however, we expect that the absolute number of events relevant for the fit will not change substantially.

$\S$ The reason for this is the presence of some tension between theoretical predictions from threedimensional fluxes and MACRO as well as Super-K thru-going muon data (see discussion in second reference of [12]). This tension did not appear with older one-dimensional fluxes, and makes it particularly important to properly take into account all the correlations between different data sets. For Super-K detailed information on the correlation between various sub-samples (sub-GeV, multi-GeV, stopping and thru-going muons) can be extracted from Ref. 37], whereas the relevant information to include MACRO is not available. Since in this analysis we are more interested in the robustness rather than in the strength of our results, we restrict our analysis to Super-K data only. A consistent way of combining Super-K and MACRO data using three-dimensional fluxes is however under investigation. 


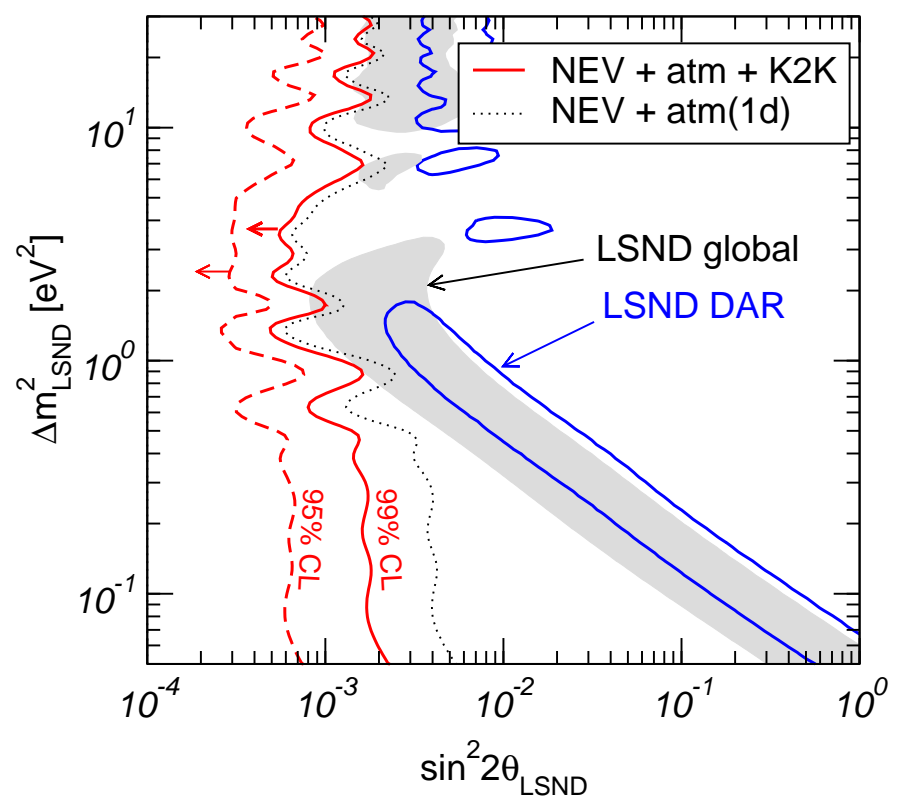

Figure 20. Upper bound on $\sin ^{2} 2 \theta_{\mathrm{LSND}}$ from NEV, atmospheric and K2K neutrino data in $(3+1)$ schemes. The bound is calculated for each $\Delta m_{\mathrm{LSND}}^{2}$ using the $\Delta \chi^{2}$ for 1 d.o.f. The dotted line corresponds to the bound at 99\% C.L. without K2K and using one-dimensional atmospheric fluxes. Also shown are the regions allowed at 99\% C.L. (2 d.o.f.) from global LSND data 113] and decay-at-rest (DAR) LSND data 128].

against a sterile component, since the detailed informations which are needed to use these data are presently not available outside the Super-K collaboration.

\section{3. $(3+1)$ : strongly disfavoured by $S B L$ data}

It is known for a long time [120, 129, 130, 131, 132, 133, 134] that $(3+1)$ mass schemes are disfavoured by the comparison of SBL disappearance data [118, 119] with the LSND result. The reason is that in $(3+1)$ schemes the relation $\sin ^{2} 2 \theta_{\mathrm{LSND}}=4 d_{e} d_{\mu}$ holds, and the parameters $d_{e}$ and $d_{\mu}$ (see Eq. (19)) are strongly constrained by $\nu_{e}$ and $\nu_{\mu}$ disappearance experiments, leading to a double suppression of the LSND amplitude. In Ref. 132] it was realized that the up-down asymmetry observed in atmospheric $\mu$ events leads to an additional constraint on $d_{\mu}$. The $\Delta \chi^{2}\left(d_{\mu}\right)$ from the fit to atmospheric $+\mathrm{K} 2 \mathrm{~K}$ data is shown in the right panel of Fig. 19. We find that the use of the new atmospheric fluxes 32] as well as K2K data [17] considerably strengthen the constraint on $d_{\mu}$ : the new bound $d_{\mu} \leq 0.065$ at $99 \%$ C.L. decreases roughly a factor 2 with respect to the previous bound $d_{\mu}<0.13$ [36, 127], as implied by fitting atmospheric data with onedimensional fluxes [39] and without K2K. Following Ref. [127] we show in Fig. 20 the upper bound on the LSND oscillation amplitude $\sin ^{2} 2 \theta_{\text {LSND }}$ from the combined analysis of NEV and atmospheric neutrino data. This figure illustrates that the improvement implied by the stronger bound on $d_{\mu}$ is mostly relevant for lower values of $\Delta m_{\mathrm{LSND}}^{2}$. From this figure we see that the bound is incompatible with the signal observed in LSND at the 95\% C.L. Only marginal overlap regions exist between the bound and global LSND data 


\begin{tabular}{|c|crrr|c|c|}
\hline & SOL & ATM & LSND & NEV & $\chi_{\mathrm{PG}}^{2}$ & PG \\
\hline$(3+1)$ & 0.0 & 0.4 & 5.7 & 10.9 & 17.0 & $1.9 \times 10^{-3}(3.1 \sigma)$ \\
$(2+2)$ & 5.3 & 20.8 & 0.6 & 7.3 & 33.9 & $7.8 \times 10^{-7}(4.9 \sigma)$ \\
\hline
\end{tabular}

Table 2. Parameter GOF and the contributions of different data sets to $\chi_{\mathrm{PG}}^{2}$ in $(3+1)$ and $(2+2)$ neutrino mass schemes.

if both are taken at 99\% C.L. Using only the decay-at-rest LSND data sample [128] the disagreement is even more severe. These results show that $(3+1)$ schemes are strongly disfavoured by SBL disappearance data.

\subsection{Comparing $(3+1),(2+2)$ and $(3+0)$ hypotheses}

Using the methods developed in Ref. 123] we perform a global fit to the oscillation data in the four-neutrino framework. This approach allows to statistically compare the different hypotheses. Let us first evaluate the GOF of $(3+1)$ and $(2+2)$ spectra using the PG method described in Ref. [125]. We divide the global oscillation data into the four data sets SOL, ATM, LSND and NEV. Then we use the PG method to test the statistical compatibility of these data sets assuming a given neutrino mass scheme. Following Ref. 121] we consider

$$
\begin{aligned}
\bar{\chi}^{2} & =\Delta \chi_{\mathrm{SOL}}^{2}\left(\theta_{\mathrm{SOL}}, \Delta m_{\mathrm{SOL}}^{2}, \eta_{s}\right)+\Delta \chi_{\mathrm{ATM}}^{2}\left(\theta_{\mathrm{ATM}}, \Delta m_{\mathrm{ATM}}^{2}, \eta_{s}, d_{\mu}\right) \\
& +\Delta \chi_{\mathrm{NEV}}^{2}\left(\theta_{\mathrm{LSND}}, \Delta m_{\mathrm{LSND}}^{2}, d_{\mu}, \eta_{e}\right)+\Delta \chi_{\mathrm{LSND}}^{2}\left(\theta_{\mathrm{LSND}}, \Delta m_{\mathrm{LSND}}^{2}\right),
\end{aligned}
$$

where $\Delta \chi_{X}^{2}=\chi_{X}^{2}-\left(\chi_{X}^{2}\right)_{\min }(X=\mathrm{SOL}, \mathrm{ATM}, \mathrm{NEV}, \mathrm{LSND})$. In Tab. 2 we show the contributions of the 4 data sets to $\chi_{\mathrm{PG}}^{2} \equiv \bar{\chi}_{\text {min }}^{2}$ for $(3+1)$ and $(2+2)$ oscillation schemes. As expected we observe that in $(3+1)$ schemes the main contribution comes from SBL data due to the tension between LSND and NEV data in these schemes. For $(2+2)$ oscillation schemes a large part of $\chi_{\mathrm{PG}}^{2}$ comes from solar and atmospheric data, due to the rejection against a sterile neutrino contribution of these two data sets, as discussed in Sec. 5.2. The contribution from NEV data in $(2+2)$ comes mainly from the tension between LSND and KARMEN [128], which does not depend on the mass scheme.

The parameter goodness of fit is obtained by evaluating $\chi_{\mathrm{PG}}^{2}$ for 4 d.o.f. [125]. This number of degrees of freedom corresponds to the 4 parameters $\eta_{s}, d_{\mu}, \theta_{\mathrm{LSND}}, \Delta m_{\mathrm{LSND}}^{2}$ describing the coupling of the different data sets (see Eq. (22) and Fig. 18). The best GOF is obtained in the $(3+1)$ case. However, even in this best case the PG is only $0.19 \%$. This PG value is slightly reduced with respect to our previous result $0.56 \%, 121,122$. because of the improved limit on $d_{\mu}$ from atmospheric + K2K data (see Sec. [5.3). The PG of $7.8 \times 10^{-7}$ for $(2+2)$ schemes shows that these mass schemes are essentially ruled out by the disagreement between the individual data sets. As mentioned in Sec. 5.2 the exclusion of $(2+2)$ schemes is slightly weaker as previously [122] since we now do not include MACRO data in our analysis.

Although we have seen that none of the four-neutrino mass schemes provides a good fit to the global oscillation data including LSND, it is interesting to consider 
the relative status of the three hypotheses $(3+1),(2+2)$ and the three-active neutrino scenario $(3+0)$. This can be done by comparing the $\chi^{2}$ value of the best fit point - which occurs for the $(3+1)$ scheme - with the ones corresponding to $(2+2)$ and $(3+0)$. First we observe that $(2+2)$ schemes are strongly disfavoured with respect to $(3+1)$ with a $\Delta \chi^{2}=16.9$. For 4 d.o.f. this is equivalent to an exclusion at $3.1 \sigma$. Furthermore, we find that $(3+0)$ is disfavoured with a $\Delta \chi^{2}=17.5$ (corresponding to $3.2 \sigma$ for 4 d.o.f.) with respect to $(3+1)$. This reflects the high statistical significance of the LSND result, since in a $(3+0)$ scheme no effect is predicted for LSND.

To summarize, we find that four-neutrino schemes do not provide a satisfactory fit to the global data. The strong rejection of non-active oscillation in the solar+KamLAND and atmospheric $+\mathrm{K} 2 \mathrm{~K}$ neutrino data rules out $(2+2)$ schemes, irrespective of whether LSND is confirmed or not. Using an improved goodness of fit method especially sensitive to the combination of data sets we find that $(2+2)$ schemes are ruled out at the $4.9 \sigma$ level. On the other hand $(3+1)$ spectra are disfavoured by the disagreement of LSND with short-baseline disappearance data, leading to a marginal GOF of $1.9 \times 10^{-3}(3.1 \sigma)$. Should LSND be confirmed it would be very desirable to have more data on $\nu_{e}$ and/or $\nu_{\mu}$ SBL disappearance to decide about the status of $(3+1)$ schemes. In that case a positive signal is predicted right at the sensitivity edge of existing experiments.

More drastic attempts to reconcile the LSND signal with the rest of neutrino oscillation data have been reviewed in Ref. 26]. For example, in Ref. 135] a fiveneutrino scheme is invoked to reconcile all the data. In Ref. [136] it has been shown that even the rather drastic assumption of CPT violation in a three-neutrino framework [137, 138] does not provide a satisfactory description of the global neutrino data set including LSND. Similarly, an interpretation of the LSND signal in terms of a non-standard muon decay is disfavoured by KARMEN [139].

We conclude that currently no convincing explanation for the LSND result exists, and it remains a puzzle how to reconcile this evidence with the rest of the data. It is therefore very important to settle this issue experimentally. A confirmation of the LSND signal by the MiniBooNE experiment [140] would be very exciting and would require some novel physics ideas.

\section{Summary and conclusions}

We have given a brief review of the status of global analyses of neutrino oscillations, taking into account the latest neutrino data, including the most recent updates of KamLAND and K2K presented at Neutrino2004, as well as state-of-the-art solar and atmospheric neutrino flux calculations. We presented two-neutrino solar + KamLAND results, as well as two-neutrino atmospheric $+\mathrm{K} 2 \mathrm{~K}$ oscillation regions, and a discussion in each case of the robustness with which the oscillation hypothesis can be established, in view of possible modifications. These might come from the assumed theoretical fluxes, the non-validity of the Standard Model neutrino interaction cross sections or the existence of non-trivial neutrino propagation properties beyond oscillations. As case 
studies we have mentioned the robustness of the solar neutrino oscillation hypothesis vis a vis the possible existence of radiative-zone solar density fluctuations, nonzero convective-zone solar magnetic fields and neutrino transition magnetic moments. For the atmospheric $+\mathrm{K} 2 \mathrm{~K}$ analysis we have considered explicitly the robustness of the oscillation hypothesis against the possible existence of flavour or universality violating non-standard neutrino interactions.

Furthermore, we have performed a fit to the most recent world neutrino data sample in the three-flavour framework. The results of this global analysis are summarized in Fig. 12 and Tab. 11, where we give the best fit values and allowed ranges of the threeflavour oscillation parameters. In addition we discussed in detail the status of the small parameters $\alpha \equiv \Delta m_{\mathrm{SOL}}^{2} / \Delta m_{\mathrm{ATM}}^{2}$ and $\sin ^{2} \theta_{13}$, which characterize the strength of $\mathrm{CP}$ violating effects in neutrino oscillations, highlighting the improvement of the bound on $\sin ^{2} \theta_{13}$ implied by the inclusion of the recent KamLAND data. Finally, we gave a review over the current status of four-neutrino interpretations of the LSND anomaly, in view of the most recent experimental and theoretical advances.

All in all, we can say beyond reasonable doubt that neutrino masses, discovered through atmospheric neutrino oscillations, have now also been confirmed in the solar neutrino oscillation channel thanks to the important input of the KamLAND experiment. Theory-wise, while the SSM was necessary in order to establish the need for physics beyond the Standard Model, it has now been made to some extent irrelevant by the high precision of the experiments which currently dominate the determination of solar neutrino oscillation parameters. The next goal in the agenda is the determination of the small parameter $\sin ^{2} \theta_{13}$ that characterizes the strength of $\mathrm{CP}$ violating effects in neutrino oscillations, and the exploration of the Majorana nature of the neutrino which will be sensitive to the other leptonic CP phases.

\section{Acknowledgments}

This review is based on work with a number of collaborators with whom we have had many (sometimes passionate) discussions. The list includes E.Kh. Akhmedov, J. Barranco, C.P. Burgess, N.S. Dzhalilov, N. Fornengo, M.C. González-García, W. Grimus, M. Guzzo, P.C. de Holanda, O. Miranda, H. Nunokawa, C. Peña-Garay, T.I. Rashba, A.I. Rez, V.B. Semikoz and R. Tomàs. This work was supported by Spanish grant BFM2002-00345, by the European Commission RTN grant HPRN-CT2000-00148 and the ESF Neutrino Astrophysics Network. M.M. is supported by the National Science Foundation grant PHY0098527. T.S. has been supported by the "Sonderforschungsbereich 375 für Astro-Teilchenphysik der Deutschen Forschungsgemeinschaft". M.T. has been supported by FPU fellowship AP2000-1953. 


\section{Appendix A. The new KamLAND results}

In this appendix we discuss in some detail the most recent data from KamLAND [15]. In Appendix A.1 we give the details of our data analysis, whereas in Appendix A.2 we discuss the physics results.

\section{Appendix A.1. Analysis details of the new data}

We have modified our previous KamLAND analysis methods [67, 68] applied to the first published KamLAND data [1] in various aspects to take into account the characteristics of the new data. First, to analyze KamLAND data one has to know the contribution of the various power reactors to the signal. We extract the relevant information from Fig. 1(b) of Ref. [15], where the no-oscillation signal is given as a function of the distance to the detector. Second, we use an improved parameterization [69] of the anti-neutrino flux emitted by the isotopes ${ }^{235} \mathrm{U},{ }^{239} \mathrm{Pu},{ }^{238} \mathrm{U}$ and ${ }^{241} \mathrm{Pu}$ in the nuclear reactors. Third, we include 2.69 accidental background events in the lowest energy bin. For the $4.8 \pm 0.9$ background events expected from the beta-decay of ${ }^{9} \mathrm{Li}$ and ${ }^{8} \mathrm{He}$ we assume regular betaspectra with endpoints of 13.6 and $10.7 \mathrm{MeV}$, respectively (see, e.g., Fig. 11 of Ref. [40]). Fourth, we include the small matter effects.

In general most information can be extracted from data by un-binned Likelihood methods (see Ref. 67] for the case of the first KamLAND data). Unfortunately it is not possible to obtain event-based energy information for the current KamLAND sample, and one has to stick with binned data outside the collaboration. Traditionally data is given in bins of equal size in the prompt energy $E_{\mathrm{pr}}$ (see Fig. 2(b) of Ref. [15]). However, it turns out that in the case of KamLAND more information can be obtained if data is binned equally in $1 / E_{\mathrm{pr}}$. The relevant information can be extracted from Fig. 3 of Ref. [15], where the ratio of the observed spectrum to the expectation for no oscillation is given in 13 bins of $180 \mathrm{~km} / E_{\nu}[\mathrm{MeV}]$. We show the data binned in $1 / E_{\mathrm{pr}}$ as well as in $E_{\mathrm{pr}}$ in Fig. A1. The $1 / E_{\mathrm{pr}}$ binning is more useful for two reasons. First, it is more natural to make the bins smaller in the region of many events (low energy) and wider in the high energy region, where there are very few events. This maintains more energy information in the region of greater statistics. Second, since the frequency of neutrino oscillations is proportional to $1 / E_{\nu}$ this binning is more appropriate for the signal we are interested.

Concerning the statistical analysis, we adopt a Poisson $\chi^{2}$-function, and make extensive use of the pull-method to implement various systematical errors. In the overall normalization uncertainty we include only the detector-specific contributions by summing up the errors of the left column of Tab. 1 from Ref. [15], which gives $\sigma_{\text {det }}=5.47 \%$. The uncertainties associated to the anti-neutrino flux are treated according to the method presented in Ref. 69]. We include an uncertainty on the thermal power $(2 \%)$ and the fuel composition $(1 \%)$ of each individual reactor, as well as the spectral uncertainty of the emitted anti-neutrino fluxes. In agreement with Ref. 69] we find that flux related uncertainties play only a minor role in the KamLAND analysis. 


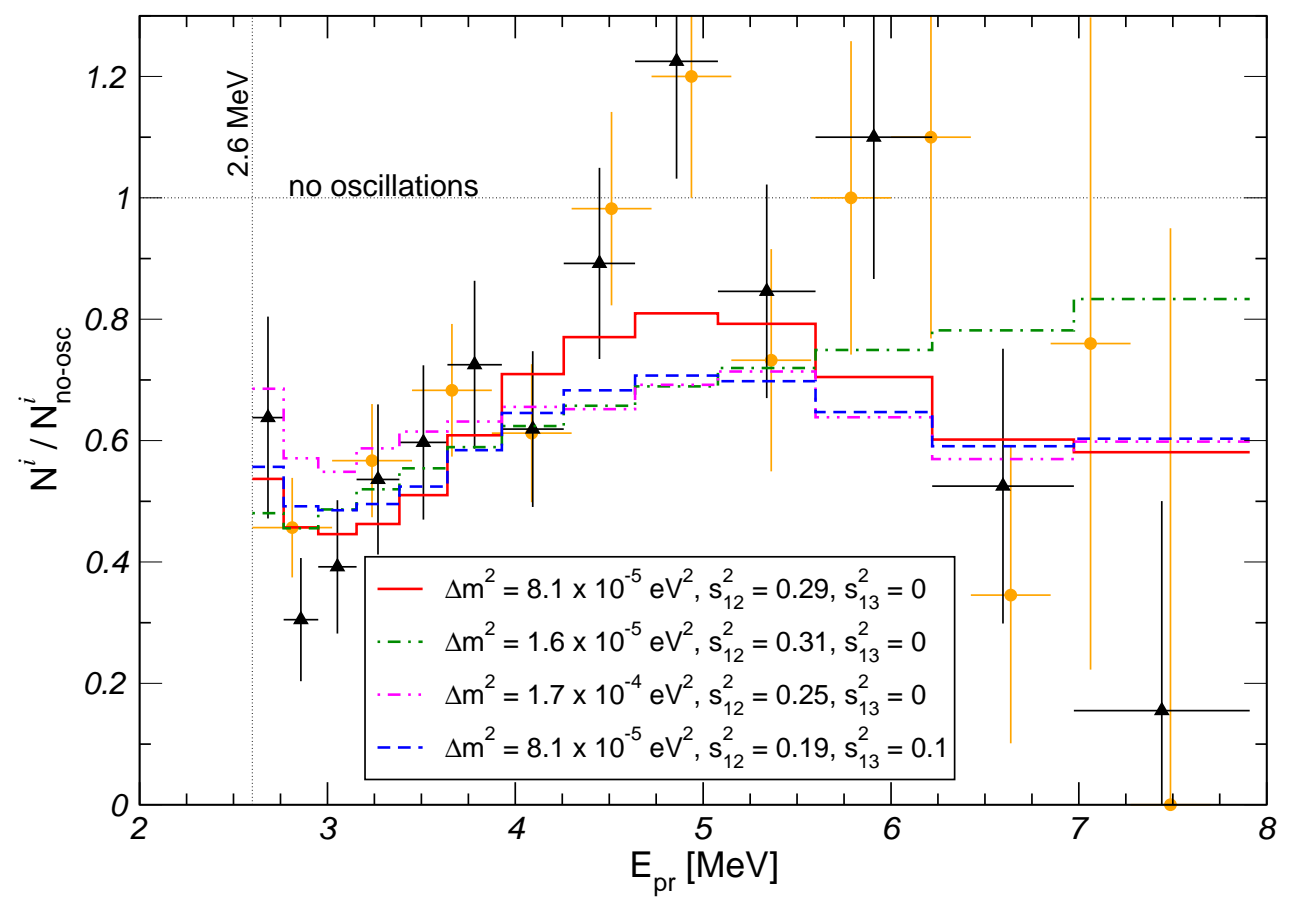

Figure A1. Data divided by the expectation for no oscillations for equal bins in $1 / E_{\mathrm{pr}}$ (triangles) and in $E_{\mathrm{pr}}$ (dots). Also shown is the spectrum for the best fit point (solid), the local best fit points in the low $\Delta m^{2}$ (dash-dotted) and high $\Delta m^{2}$ (dash-dot-dotted) regions, and for a large value of $\sin ^{2} \theta_{13}$ (dashed).

\section{Appendix A.2. Discussion of the KamLAND results}

In Fig. A1 we show the predictions for the probabilities in the various bins $P_{i} \equiv$ $N_{\text {osc }}^{i} / N_{\text {no-osc }}^{i}$ compared to the data $P_{i}^{\text {obs }} \equiv N_{\text {obs }}^{i} / N_{\text {no-osc }}^{i}$. From this figure one can see that the probabilities are rather low for low energies, whereas in the region around 4.5 MeV even slightly more events than expected for no oscillations have been observed. This is a very characteristic pattern indicating rather strong spectral distortion, and only the best fit parameters (solid line) can reproduce this shape. All other parameter choices shown in the figure have problems to accommodate the high data points in the middle of the spectrum. This holds for the solution around $\Delta m^{2} \simeq 1.6 \times 10^{-5} \mathrm{eV}^{2}$ as well as for the high-LMA solution at $\Delta m^{2} \simeq 1.7 \times 10^{-4} \mathrm{eV}^{2}$ (compare Fig. [6). The rejection power to these two "solutions" is significantly increased by the $1 / E_{\mathrm{pr}}$ binning with respect to the $E_{\mathrm{pr}}$ binning: In our analysis we obtain a $\Delta \chi^{2}=7.5(11.3)$ for the local minimum at $1.6(17) \times 10^{-5} \mathrm{eV}^{2}$, whereas with the traditional $E_{\mathrm{pr}}$ binning we find $\Delta \chi^{2}=4.0(8.5)$. In particular, to disfavour the high-LMA region the more precise energy information at low energies provided by the $1 / E_{\mathrm{pr}}$ bins is crucial, whereas for the low region the high energy part seems to be important.

Let us now discuss the bound on $\sin ^{2} \theta_{13}$ from the KamLAND data. From Fig. A1 one can see that turning on $\sin ^{2} \theta_{13}$ leads to a flatter energy spectrum and it gets more difficult to accommodate the low probabilities for low energies simultaneously with the high data points in the middle part of the spectrum. It is also clear from the relevant 

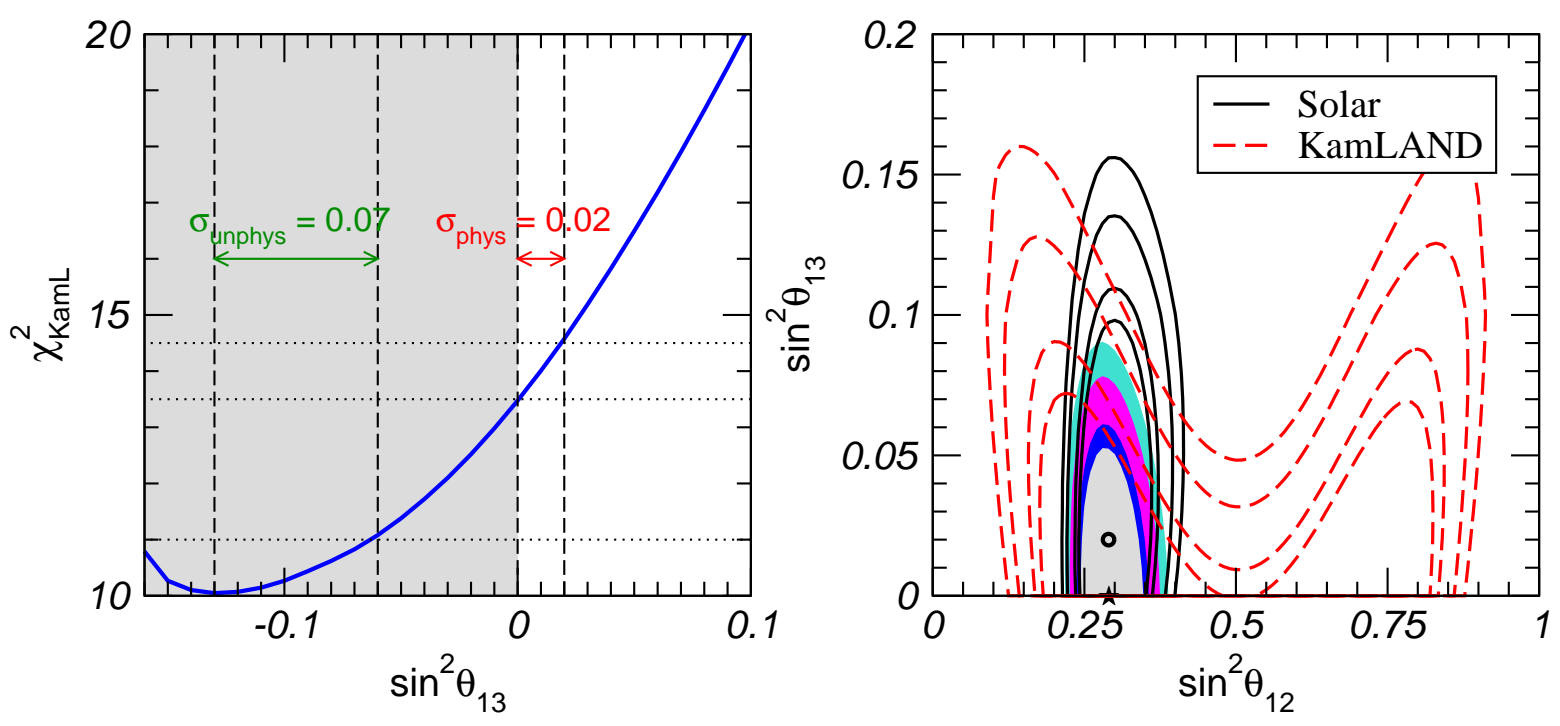

Figure A2. Left panel: $\chi^{2}$ from KamLAND data as a function of $\sin ^{2} \theta_{13}$ including the unphysical region $\sin ^{2} \theta_{13}<0$. Right panel: allowed regions at $90 \%, 95 \%, 99 \%$ and $3 \sigma$ of $\sin ^{2} \theta_{13}$ and $\sin ^{2} \theta_{12}\left(2\right.$ d.o.f.) for fixed $\Delta m_{\mathrm{SOL}}^{2}=8.1 \times 10^{-5} \mathrm{eV}^{2}$ from KamLAND data (dashed), solar data (solid), and KamLAND+solar data combined (shaded regions).

three-flavour survival probability in vacuum

$$
P_{e e}=1-\frac{1}{2} \sin ^{2} 2 \theta_{13}-\cos ^{4} \theta_{13} \sin ^{2} 2 \theta_{12} \sin ^{2} \frac{\Delta m_{21}^{2} L}{4 E_{\nu}}
$$

that values of $\sin ^{2} \theta_{13}>0$ suppress the oscillatory term. In fact, if a fit to the KamLAND data is performed without imposing the constraint $\sin ^{2} \theta_{13} \geq 0$ one finds a best fit point within the unphysical region at $\sin ^{2} \theta_{13}=-0.13$ (see Fig. A2). Because of the rather large difference in the probability between the low and medium energy bins the fit is improved by a $\Delta \chi^{2} \simeq 3.5$ by allowing an enhancement of the oscillatory term in Eq. (A.1) due to values of $\cos ^{4} \theta_{13}>1$, i.e., $\sin ^{2} \theta_{13}<0$. Fig. A2 illustrates that this fact leads to a rather strong bound on $\sin ^{2} \theta_{13}$ if the analysis is restricted to the physical region. Just from the statistical power of the data one would expect a $1 \sigma$ error on $\sin ^{2} \theta_{13}$ of $\sigma \simeq 0.07$. However, due to the particular fluctuation observed in the actual data the best fit point lies in the unphysical region. This implies that for $\sin ^{2} \theta_{13}=0$ there is already some tension in the fit, and a significantly smaller error of $\sigma=0.02$ is obtained within the physical region.\|

Finally, in the right panel of Fig. A2 we illustrate, why the combination of KamLAND and solar data leads to a further notable improvement of the bound on $\sin ^{2} \theta_{13}$. From Eq. (A.1) one expects for KamLAND a correlation between $\sin ^{2} \theta_{13}$ and $\sin ^{2} 2 \theta_{12}$. Solving Eq. (A.1) for $\sin ^{2} 2 \theta_{12}$ and expanding up to first order in $\sin ^{2} \theta_{13}$ one $\|$ We note that in such a case a reliable bound can be calculated by performing a Monte Carlo simulation of many synthetic data sets. This is beyond the scope of the present article, and we throughout use the naive method of calculating bounds by considering $\Delta \chi^{2}$-values as implied by Gaussian statistics and restricting the analysis to the physical region. 


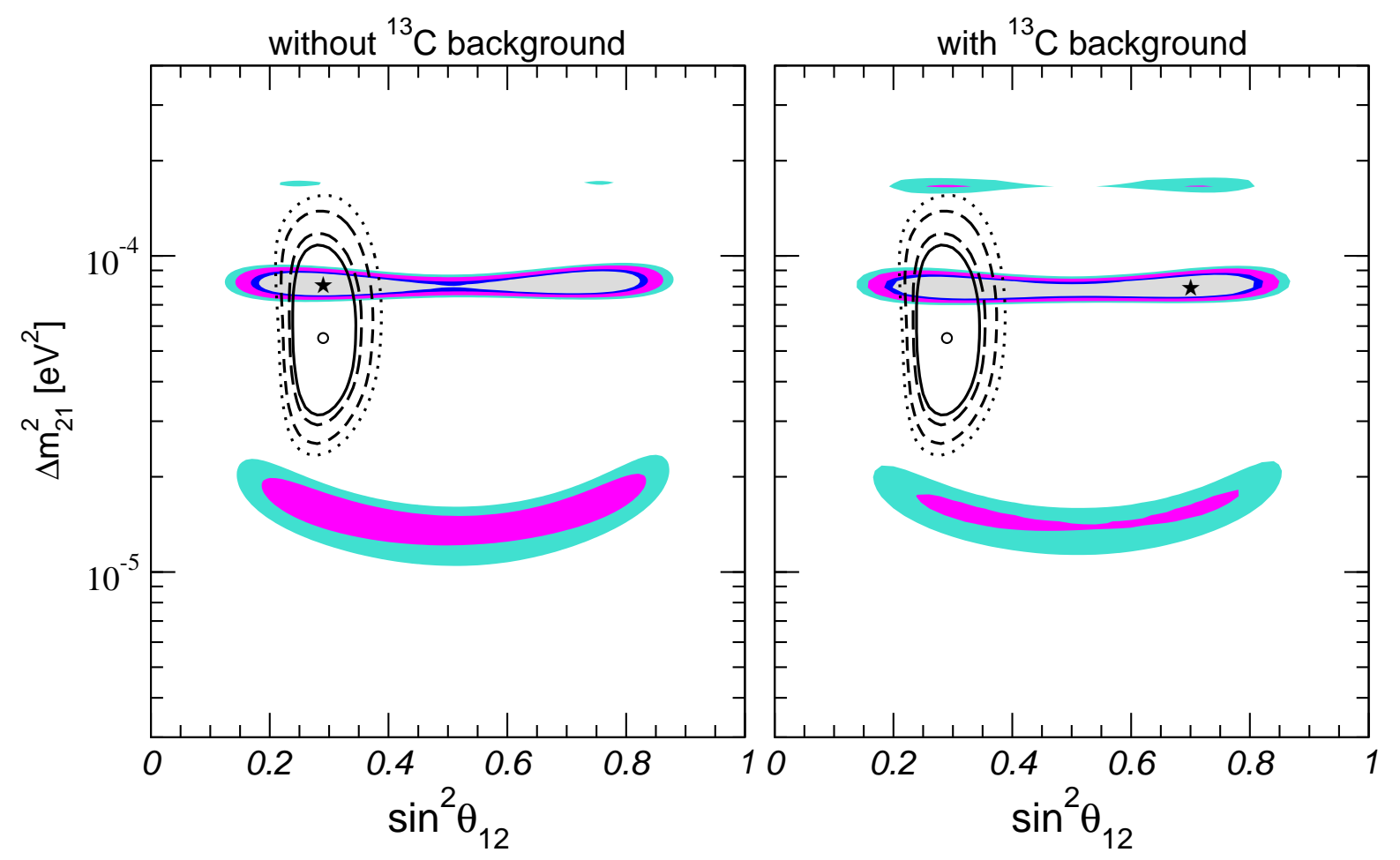

Figure B1. Allowed $\left(\sin ^{2} \theta_{\mathrm{SOL}}, \Delta m_{\mathrm{SOL}}^{2}\right)$ regions at $90 \%, 95 \%, 99 \%$, and $3 \sigma$ C.L. for 2 d.o.f. The left panel corresponds to Fig. [6] whereas in the right panel the new background from ${ }^{13} \mathrm{C}$ has been taken into account.

finds

$$
\sin ^{2} 2 \theta_{12} \sin ^{2} \frac{\Delta m_{21}^{2} L}{4 E_{\nu}} \simeq 1-P_{e e}-2 P_{e e} \sin ^{2} \theta_{13}
$$

For a given survival probability Eq. A.2 implies a negative correlation between $\sin ^{2} \theta_{13}$ and $\sin ^{2} 2 \theta_{12}$. This trend is visible also in the actual fit, see Fig. A2, where the allowed region for these two parameters is shown for fixed $\Delta m^{2}$. One observes that increasing $\sin ^{2} \theta_{13}$ can be compensated to some extent by decreasing $\sin ^{2} \theta_{12}$ (within the "light side" $\left.\sin ^{2} \theta_{12}<0.5\right)$. This, however, is in disagreement with solar data, which provide a stable lower bound on $\sin ^{2} \theta_{12}$ due to the fundamentally different conversion mechanism (MSW matter effect in the sun versus vacuum oscillations). The combined analysis of KamLAND and solar data leads essentially to the intersection of the two individual allowed regions, which further improves the bound on $\sin ^{2} \theta_{13}$.

\section{Appendix B. Implications of a new background in KamLAND}

After this paper has been published it was realised that a background from the reaction ${ }^{13} \mathrm{C}(\alpha, n){ }^{16} \mathrm{O}$ contributes to the KamLAND data, which has not been taken into account in the first version of Ref. [15], on which our analysis is based. In this appendix we show that the impact of this new background on our results is small, and we present the updated results, where the changes are noticeable. 

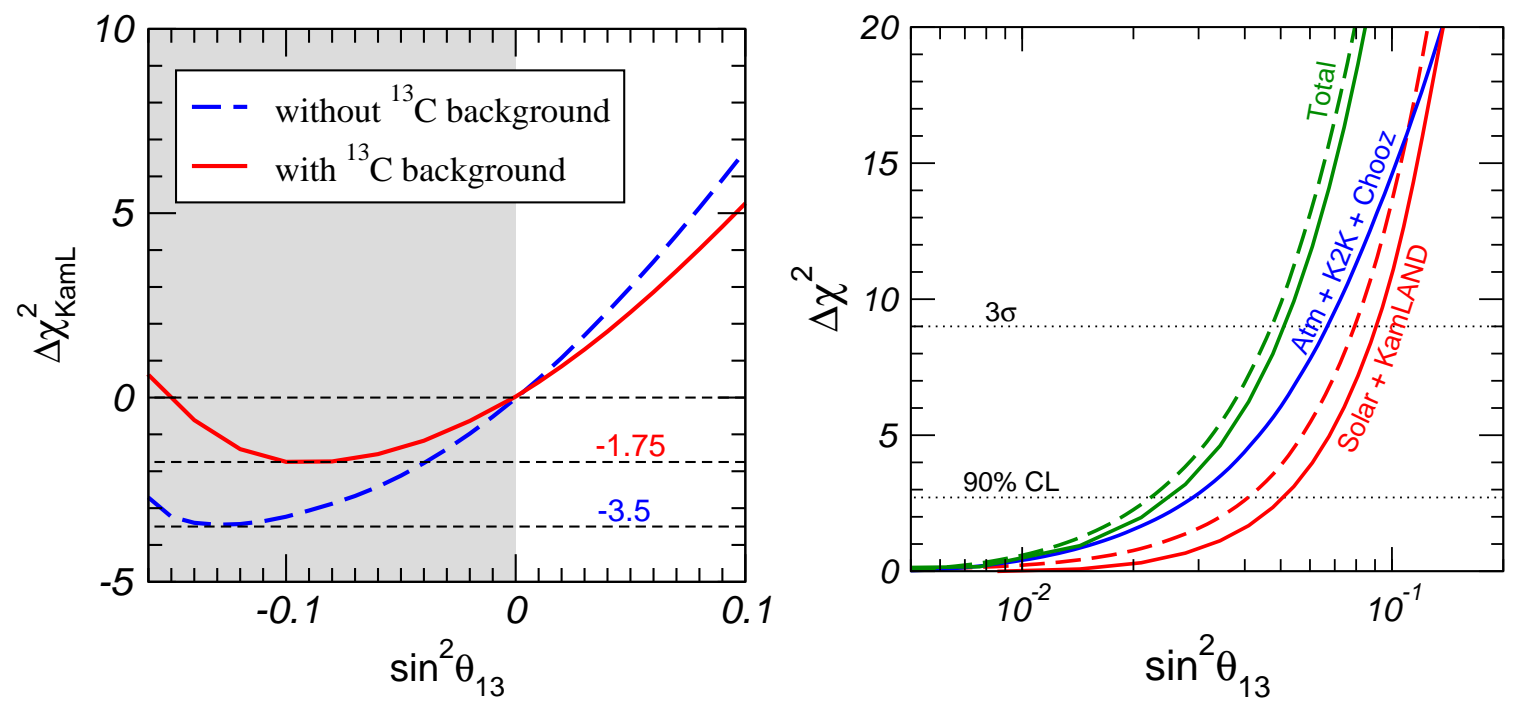

Figure B2. Left panel: $\Delta \chi^{2}$ from KamLAND data allowing for negative values of $\sin ^{2} \theta_{13}$ with and without accounting for the ${ }^{13} \mathrm{C}$ background. Right panel: $\Delta \chi^{2}$ as a function of $\sin ^{2} \theta_{13}$ for various data samples. The ${ }^{13} \mathrm{C}$ background is (is not) taken into account for the solid (dashed) curves.

The reaction ${ }^{13} \mathrm{C}(\alpha, n){ }^{16} \mathrm{O}$ leads to $10.3 \pm 7.1$ events above the $2.6 \mathrm{MeV}$ threshold in KamLAND, and hence the total background is increased to $17.8 \pm 7.3$ events. This new background is mainly concentrated around $6 \mathrm{MeV}$, and the main effect of subtracting these events from the reactor data is that the relatively high data point at $6 \mathrm{MeV}$ visible in Fig. A1 is moved from a value of 1.1 to 0.8 , in better agreement with the prediction for oscillation. Indeed, we now find $\chi_{\min }^{2}=9.5$ instead of 13.5 , i.e. the quality of the fit improves. The impact of the new background on the determination of the solar parameters is shown in Fig. B1. The best fit point for $\Delta m_{\mathrm{SOL}}^{2}$ moves from $8.1 \times 10^{-5} \mathrm{eV}^{2}$ to $7.9 \times 10^{-5} \mathrm{eV}^{2}$. However, the allowed region around the best fit point is very stable. The fact that the best fit point occurs now for $\sin ^{2} \theta_{\text {SOL }}>0.5$ has no statistical significance. The low- $\Delta m_{\mathrm{SOL}}^{2}$ solution is slightly more disfavoured, with $\Delta \chi^{2}=8.6$ instead of 7.4 , whereas the the high- $\Delta m_{\mathrm{SOL}}^{2}$ solution becomes somewhat better, with $\Delta \chi^{2}=8.8$ instead of 11.2 . Since these solutions are ruled out anyway by solar data, the overall changes in the global fit are very small.

The impact of the new background on the bound on $\theta_{13}$ is illustrated in Fig. B2, The left panel shows the $\chi^{2}$ of KamLAND data without imposing the constraint $\sin ^{2} \theta_{13} \geq 0$. We observe that the best fit occurs now closer to the physical region, and the $\Delta \chi^{2}$ between $\sin ^{2} \theta_{13}=0$ and the best fit point decreases from 3.5 to 1.75 . This again shows that the quality of the fit improves. As a consequence the bound on $\sin ^{2} \theta_{13}$ from KamLAND (constraining the fit to the physical region) becomes slightly weaker. From the right panel one can see that the impact on the bound from global data is rather small. Finally, in Tab. B1 we update the results of the global three-neutrino analysis given in Tab. 1, taking into account the new background in KamLAND data. Only the numbers for $\Delta m_{\mathrm{SOL}}^{2}$ and $\sin ^{2} \theta_{13}$ change slightly. 


\begin{tabular}{|l|c|c|c|c|}
\hline parameter & best fit & $2 \sigma$ & $3 \sigma$ & $4 \sigma$ \\
\hline$\Delta m_{21}^{2}\left[10^{-5} \mathrm{eV}^{2}\right]$ & 7.9 & $7.3-8.5$ & $7.1-8.9$ & $6.8-9.3$ \\
$\Delta m_{31}^{2}\left[10^{-3} \mathrm{eV}^{2}\right]$ & 2.2 & $1.7-2.9$ & $1.4-3.3$ & $1.1-3.7$ \\
$\sin ^{2} \theta_{12}$ & 0.30 & $0.25-0.34$ & $0.23-0.38$ & $0.21-0.41$ \\
$\sin ^{2} \theta_{23}$ & 0.50 & $0.38-0.64$ & $0.34-0.68$ & $0.30-0.72$ \\
$\sin ^{2} \theta_{13}$ & 0.000 & $\leq 0.031$ & $\leq 0.051$ & $\leq 0.073$ \\
\hline
\end{tabular}

Table B1. Update of Tab. 1 taking into account the background from ${ }^{13} \mathrm{C}$ in KamLAND: Best-fit values, $2 \sigma, 3 \sigma$, and $4 \sigma$ intervals (1 d.o.f.) for the three-flavour neutrino oscillation parameters from global data including solar, atmospheric, reactor (KamLAND and CHOOZ) and accelerator (K2K) experiments.

\section{References}

[1] R. Davis, Prog. Part. Nucl. Phys. 32, 13 (1994).

[2] B. T. Cleveland et al., Astrophys. J. 496, 505 (1998).

[3] SAGE Collaboration, J. N. Abdurashitov et al., Phys. Rev. C60, 055801 (1999), astro-ph/9907113.

[4] GNO Collaboration, Nucl. Phys. Proc. Suppl. 110, 311 (2002).

[5] GALLEX Collaboration, W. Hampel et al., Phys. Lett. B447, 127 (1999).

[6] GNO Collaboration, M. Altmann et al., Phys. Lett. B490, 16 (2000), hep-ex/0006034.

[7] Super-Kamiokande Collaboration, S. Fukuda et al., Phys. Lett. B539, 179 (2002), hep-ex/0205075.

[8] SNO Collaboration, Q. R. Ahmad et al., Phys. Rev. Lett. 89, 011301 (2002), nucl-ex/0204008.

[9] SNO Collaboration, Q. R. Ahmad et al., Phys. Rev. Lett. 89, 011302 (2002), nucl-ex/0204009.

[10] SNO Collaboration, S. N. Ahmed et al., Phys. Rev. Lett. , 041801 (2003), nucl-ex/0309004.

[11] Super-Kamiokande Collaboration, Y. Fukuda et al., Phys. Rev. Lett. 81, 1562 (1998), hep-ex/9807003.

[12] MACRO Collaboration, A. Surdo, Nucl. Phys. Proc. Suppl. 110, 342 (2002); G. Giacomelli and A. Margiotta, Phys. Atom. Nucl. 67, 1139 (2004), hep-ex/0407023.

[13] Soudan 2 Collaboration, M. Sanchez et al., Phys. Rev. D68, 113004 (2003), hep-ex/0307069.

[14] KamLAND Collaboration, K. Eguchi et al., Phys. Rev. Lett. 90, 021802 (2003), hep-ex/0212021. 
[15] KamLAND Collaboration, T. Araki et al., hep-ex/0406035; Talk by G. Gratta at Neutrino 2004, 14-19 June 2004, Paris, http://neutrino2004.in2p3.fr/

[16] K2K Collaboration, M. H. Ahn et al., Phys. Rev. Lett. 90, 041801 (2003), hep-ex/0212007.

[17] T. Nakaya, Talk at Neutrino 2004, 14-19 June 2004, Paris, http://neutrino2004.in2p3.fr/

[18] M. Gell-Mann, P. Ramond and R. Slansky, (1979), Print-80-0576 (CERN).

[19] T. Yanagida, (KEK lectures, 1979), ed. Sawada and Sugamoto (KEK, 1979).

[20] J. Schechter and J. W. F. Valle, Phys. Rev. D22, 2227 (1980).

[21] R. N. Mohapatra and G. Senjanovic, Phys. Rev. D23, 165 (1981).

[22] For a short review see J. W. F. Valle, "Neutrino masses twenty-five years later," AIP Conf. Proc. 687 (2003) 16 hep-ph/0307192.

[23] See, for example, M. Goodman's Neutrino Oscillation Industry Web-Page, http://neutrinooscillation.org/

[24] S. P. Mikheev and A. Y. Smirnov, Sov. J. Nucl. Phys. 42, 913 (1985).

[25] L. Wolfenstein, Phys. Rev. D17, 2369 (1978).

[26] S. Pakvasa and J. W. F. Valle, Proceedings of the Indian National Academy of Sciences, Part A: Vol. 70A, No. 1, p.189 - 222 (2004), Eds. D. Indumathi, M.V.N. Murthy and G. Rajasekaran hep-ph/0301061.

[27] V. Barger, D. Marfatia and K. Whisnant, Int. J. Mod. Phys. E 12 (2003) 56.

[28] M. C. Gonzalez-Garcia and Y. Nir, Rev. Mod. Phys. 75 (2003) 34.

[29] G. L. Fogli et al., Proc. of International Workshop on Astroparticle and High Energy Physics, October 14 - 18, 2003, Valencia, Spain, published at JHEP, PRHEP-AHEP2003/071, accessible from http://ific.uv.es/ahep/.

[30] M. Maltoni, T. Schwetz, M. A. Tortola and J. W. F. Valle, Phys. Rev. D68, 113010 (2003), hep-ph/0309130.

[31] J. N. Bahcall and M. H. Pinsonneault, Phys. Rev. Lett. 93, 121301 (2004), astro-ph/0402114.

[32] M. Honda, T. Kajita, K. Kasahara and S. Midorikawa, astro-ph/0404457.

[33] Super-Kamiokande Collaboration, Y. Ashie, hep-ex/0404034.

[34] Y. Hayato, http://eps2003.physik.rwth-aachen.de,

[35] M. Gonzalez-Garcia, M. Maltoni, C. Pena-Garay and J. W. F. Valle, Phys. Rev. D63, 033005 (2001), hep-ph/0009350.

[36] M. Maltoni, T. Schwetz, M. A. Tortola and J. W. F. Valle, Phys. Rev. D67, 013011 (2003), hep-ph/0207227 version v3].

[37] J. Kameda, PhD Thesis, University of Tokyo, 2002, available from the Web-page http://www-sk.icrr.u-tokyo.ac.jp/doc/sk/pub/. 
[38] G. L. Fogli, E. Lisi, A. Marrone and D. Montanino, Phys. Rev. D67, 093006 (2003), hep-ph/0303064.

[39] G. Barr, T. K. Gaisser and T. Stanev, Phys. Rev. D39, 3532 (1989).

[40] P. Huber, M. Lindner, M. Rolinec, T. Schwetz and W. Winter, hep-ph/0403068.

[41] J. Schechter and J. W. F. Valle, Phys. Rev. D25, 774 (1982); J. W. F. Valle, Phys. Lett. B131, 87 (1983); G. B. Gelmini and J. W. F. Valle, Phys. Lett. B142, 181 (1984); M. C. Gonzalez-Garcia and J. W. F. Valle, Phys. Lett. B216, 360 (1989).

[42] S. Pakvasa and K. Tennakone, Phys. Rev. Lett. 28, 1415 (1972).

[43] A. Zee, Phys. Lett. B93, 389 (1980).

[44] K. S. Babu, Phys. Lett. B203, 132 (1988).

[45] R. N. Mohapatra and J. W. F. Valle, Phys. Rev. D34, 1642 (1986).

[46] L. J. Hall, V. A. Kostelecky and S. Raby, Nucl. Phys. B267, 415 (1986).

[47] J. Bernabeu et al., Phys. Lett. B187, 303 (1987).

[48] G. C. Branco, M. N. Rebelo and J. W. F. Valle, Phys. Lett. B225, 385 (1989).

[49] N. Rius and J. W. F. Valle, Phys. Lett. B246, 249 (1990).

[50] N. Fornengo et al., Phys. Rev. D65, 013010 (2002), hep-ph/0108043.

[51] J. W. F. Valle, Prog. Part. Nucl. Phys. 26, 91 (1991).

[52] M. Hirsch and J. W. F. Valle, hep-ph/0405015.

[53] M. C. Gonzalez-Garcia and M. Maltoni, hep-ph/0404085, to appear in PRD.

[54] H. Nunokawa, Y. Z. Qian, A. Rossi and J. W. F. Valle, Phys. Rev. D54, 4356 (1996), hep-ph/9605301.

[55] G. L. Fogli, E. Lisi, A. Mirizzi and D. Montanino, Phys. Rev. D66, 013009 (2002), hep-ph/0202269.

[56] D. Grasso, H. Nunokawa and J. W. F. Valle, Phys. Rev. Lett. 81, 2412 (1998), astro-ph/9803002.

[57] A. McDonald, this volume.

[58] J. N. Bahcall and C. Pena-Garay, hep-ph/0404061.

[59] E. Bellotti and V. Gavrin, http://mocha.phys.washington.edu/.

[60] SAGE Collaboration, J. N. Abdurashitov et al., J. Exp. Theor. Phys. 95, 181 (2002), astro-ph/0204245. The latest number was presented by C. M. Cattadori, Talk at Neutrino 2004, 14-19 June 2004, Paris, http://neutrino2004.in2p3.fr/

[61] G. L. Fogli, E. Lisi, A. Marrone, D. Montanino and A. Palazzo, Phys. Rev. D66, 053010 (2002), hep-ph/0206162.

[62] A. B. Balantekin and H. Yuksel, Phys. Rev. D68, 113002 (2003), hep-ph/0309079.

[63] J. N. Bahcall, M. H. Pinsonneault and S. Basu, Astrophys. J. 555, 990 (2001), astro-ph/0010346. 
[64] P. Chankowski, A. Ioannisian, S. Pokorski and J. W. F. Valle, Phys. Rev. Lett. 86, 3488 (2001), hep-ph/0011150.

[65] G. Fiorentini, T. Lasserre, M. Lissia, B. Ricci and S. Schonert, Phys. Lett. B558, 15 (2003), hep-ph/0301042.

[66] H. Nunokawa, W. J. C. Teves and R. Zukanovich Funchal, JHEP 11, 020 (2003), hep-ph/0308175.

[67] T. Schwetz, Phys. Lett. B577, 120 (2003), hep-ph/0308003.

[68] M. Maltoni, T. Schwetz and J. W. F. Valle, Phys. Rev. D67, 093003 (2003), hep-ph/0212129.

[69] P. Huber and T. Schwetz, hep-ph/0407026.

[70] Super-Kamiokande Collaboration, M. B. Smy et al., Phys. Rev. D69, 011104 (2004), hep-ex/0309011.

[71] A. B. Balantekin, J. M. Fetter and F. N. Loreti, Phys. Rev. D54, 3941 (1996), astro-ph/9604061.

[72] H. Nunokawa, A. Rossi, V. B. Semikoz and J. W. F. Valle, Nucl. Phys. B472, 495 (1996), hep-ph/9602307.

[73] P. Bamert, C. P. Burgess and D. Michaud, Nucl. Phys. B513, 319 (1998), hep-ph/9707542.

[74] V. Castellani, S. Degl'Innocenti, W. A. Dziembowski, G. Fiorentini and B. Ricci, Nucl. Phys. Proc. Suppl. 70, 301 (1999), astro-ph/9712174.

[75] J. Christensen-Dalsgaard, Rev. Mod. Phys. 74, 1073 (2003), astro-ph/0207403.

[76] C. P. Burgess et al., Mon. Not. Roy. Astron. Soc. 348, 609 (2004), astro-ph/0304462.

[77] C. Burgess et al., Astrophys. J. 588, L65 (2003), hep-ph/0209094.

[78] C. P. Burgess et al., JCAP 0401, 007 (2004), hep-ph/0310366.

[79] K. Fujikawa and R. Shrock, Phys. Rev. Lett. 45, 963 (1980).

[80] J. Schechter and J. W. F. Valle, Phys. Rev. D24, 1883 (1981), Err. Phys. Rev. D25, 283 (1982).

[81] E. K. Akhmedov, Phys. Lett. B213, 64 (1988).

[82] C.-S. Lim and W. J. Marciano, Phys. Rev. D37, 1368 (1988).

[83] O. G. Miranda, T. I. Rashba, A. I. Rez and J. W. F. Valle, hep-ph/0311014.

[84] V. A. Kutvitskii and L. S. Solov'ev, J. Exp. Theor. Phys. 78, 456 (1994).

[85] O. G. Miranda et al., Nucl. Phys. B595, 360 (2001), hep-ph/0005259.

[86] KamLAND Collaboration, K. Eguchi et al., Phys. Rev. Lett. 92, 071301 (2004), hep-ex/0310047.

[87] Super-Kamiokande Collaboration, Y. Gando et al., Phys. Rev. Lett. 90, 171302 (2003), hep-ex/0212067.

[88] W. Grimus et al., Nucl. Phys. B648, 376 (2003), hep-ph/0208132. 
[89] J. F. Beacom and P. Vogel, Phys. Rev. Lett. 83, 5222 (1999), hep-ph/9907383.

[90] A. S. Joshipura and S. Mohanty, Phys. Rev. D66, 012003 (2002), hep-ph/0204305.

[91] J. A. Grifols, E. Masso and S. Mohanty, hep-ph/0401144.

[92] Super-Kamiokande Collaboration, D. W. Liu et al., hep-ex/0402015.

[93] A. V. Derbin, Phys. Atom. Nucl. 57, 222 (1994).

[94] TEXONO Collaboration, H. B. Li et al., hep-ex/0212003.

[95] MUNU Collaboration, Z. Daraktchieva et al., Phys. Lett. B564, 190 (2003), hep-ex/0304011.

[96] D. Y. Bardin, S. M. Bilenky and B. Pontecorvo, Phys. Lett. B32, 68 (1970).

[97] A. V. Kyuldjiev, Nucl. Phys. B243, 387 (1984).

[98] W. Grimus and T. Schwetz, Nucl. Phys. B587, 45 (2000), hep-ph/0006028.

[99] Borexino Collaboration, G. Alimonti et al., Astropart. Phys. 16, 205 (2002), hep-ex/0012030.

[100] G. L. Fogli et al., Phys. Rev. D67, 073002 (2003), hep-ph/0212127.

[101] M. C. Gonzalez-Garcia and C. Pena-Garay, hep-ph/0306001.

[102] Particle Data Group, K. Hagiwara et al., Phys. Rev. D66, 010001 (2002).

[103] M. C. Gonzalez-Garcia and M. Maltoni, Eur. Phys. J. C26, 417 (2003), hep-ph/0202218.

[104] M. Doi, T. Kotani, H. Nishiura, K. Okuda and E. Takasugi, Phys. Lett. B102, $323(1981)$.

[105] CHOOZ Collaboration, M. Apollonio et al., Phys. Lett. B466, 415 (1999), hep-ex/9907037.

[106] J. Schechter and J. W. F. Valle, Phys. Rev. D21, 309 (1980).

[107] M. Freund, Phys. Rev. D64, 053003 (2001), hep-ph/0103300.

[108] E. K. Akhmedov, R. Johansson, M. Lindner, T. Ohlsson and T. Schwetz, hep-ph/0402175.

[109] Palo Verde Collaboration, F. Boehm et al., Phys. Rev. D64, 112001 (2001), hep-ex/0107009.

[110] E. K. Akhmedov, M. A. Tortola and J. W. F. Valle, hep-ph/0404083, JHEP in press.

[111] M. Blennow, T. Ohlsson and H. Snellman, Phys. Rev. D69, 073006 (2004), hep-ph/0311098.

[112] C. Yanagisawa, Proc. of International Workshop on Astroparticle and High Energy Physics, October 14 - 18, 2003, Valencia, Spain, published at JHEP, PRHEPAHEP2003/062, accessible from http://ific.uv.es/ahep/.

[113] LSND Collaboration, A. Aguilar et al., Phys. Rev. D64, 112007 (2001), hep-ex/0104049. 
[114] J. T. Peltoniemi and J. W. F. Valle, Nucl. Phys. B406, 409 (1993), hep-ph/9302316.

[115] J. T. Peltoniemi, D. Tommasini and J. W. F. Valle, Phys. Lett. B298, 383 (1993).

[116] D. O. Caldwell and R. N. Mohapatra, Phys. Rev. D48, 3259 (1993).

[117] KARMEN Collaboration, B. Armbruster et al., Phys. Rev. D65, 112001 (2002), hep-ex/0203021.

[118] CDHS Collaboration, F. Dydak et al., Phys. Lett. B134, 281 (1984).

[119] Y. Declais et al., Nucl. Phys. B434, 503 (1995).

[120] W. Grimus and T. Schwetz, Eur. Phys. J. C20, 1 (2001), hep-ph/0102252.

[121] M. Maltoni, T. Schwetz, M. A. Tortola and J. W. F. Valle, Nucl. Phys. B643, 321 (2002), hep-ph/0207157.

[122] T. Schwetz, hep-ph/0311217.

[123] M. Maltoni, T. Schwetz and J. W. F. Valle, Phys. Rev. D65, 093004 (2002), hep-ph/0112103.

[124] M. C. Gonzalez-Garcia, M. Maltoni and C. Pena-Garay, Phys. Rev. D64, 093001 (2001), hep-ph/0105269.

[125] M. Maltoni and T. Schwetz, Phys. Rev. D68, 033020 (2003), hep-ph/0304176.

[126] H. Paes, L.-g. Song and T. J. Weiler, Phys. Rev. D67, 073019 (2003), hep-ph/0209373.

[127] M. Maltoni, T. Schwetz and J. W. F. Valle, Phys. Lett. B518, 252 (2001), hep-ph/0107150.

[128] E. D. Church, K. Eitel, G. B. Mills and M. Steidl, Phys. Rev. D66, 013001 (2002), hep-ex/0203023.

[129] S. M. Bilenky, C. Giunti and W. Grimus, Eur. Phys. J. C1, 247 (1998), hep-ph/9607372.

[130] N. Okada and O. Yasuda, Int. J. Mod. Phys. A12, 3669 (1997), hep-ph/9606411.

[131] V. D. Barger, S. Pakvasa, T. J. Weiler and K. Whisnant, Phys. Rev. D58, 093016 (1998), hep-ph/9806328.

[132] S. M. Bilenky, C. Giunti, W. Grimus and T. Schwetz, Phys. Rev. D60, 073007 (1999), hep-ph/9903454.

[133] O. L. G. Peres and A. Y. Smirnov, Nucl. Phys. B599, 3 (2001), hep-ph/0011054.

[134] C. Giunti and M. Laveder, JHEP 02, 001 (2001), hep-ph/0010009.

[135] M. Sorel, J. M. Conrad and M. Shaevitz, hep-ph/0305255.

[136] M. C. Gonzalez-Garcia, M. Maltoni and T. Schwetz, Phys. Rev. D68, 053007 (2003), hep-ph/0306226.

[137] H. Murayama and T. Yanagida, Phys. Lett. B520, 263 (2001), hep-ph/0010178.

[138] G. Barenboim, L. Borissov and J. Lykken, hep-ph/0212116.

[139] B. Armbruster et al., Phys. Rev. Lett. 90, 181804 (2003), hep-ex/0302017. 
[140] BooNE Collaboration, E. D. Zimmerman, eConf C0209101, TH05 (2002), hep-ex/0211039. 